\title{
UNKONVENTIONELLE POLITISCHE PARTIZIPATION IM ZEITVERLAUF
}

Hat die Bildungsexpansion zu einer politischen Mobilisierung beigetragen?

\author{
Andreas Hadjar und Rolf Becker
}

\begin{abstract}
Zusammenfassung: In Demokratien wird von vielen Seiten eine zunehmende Abkehr der Bürger und Bürgerinnen von Politik beklagt. Haben die Bildungsreformen der 1960er Jahre, zu deren Grundanliegen auch die Förderung politischen Engagements und die Erziehung zu mündigen Bürgerinnen und Bürgern gehörten, in diesem Punkt versagt? Im Rahmen dieses Beitrags wird der Frage nachgegangen, ob die Bildungsexpansion eine politische Mobilisierung zur Folge hatte, im Zuge derer unkonventionelle politische Partizipationsformen an Bedeutung gewonnen haben. Unter Nutzung kumulierter ALLBUS-Datensätze (1988-2000) werden zunächst soziale Hintergrundmechanismen des Zusammenhangs zwischen Bildung und politischer Partizipation auf der individuellen Ebene betrachtet, um dann anhand einer simultanen Modellierung von Alters-, Periodenund Kohorteneffekt unkonventionelle politische Partizipation im Zeitverlauf zu untersuchen. Im Ergebnis zeigen sich gleich bleibend starke Effekte des Bildungsniveaus sowie Hinweise, dass eine politische Mobilisierung stattgefunden hat. Während die höher Gebildeten sich als die hauptsächlichen Träger dieses Prozesses erweisen, bleibt die Distinktion der niedrigen Bildungsgruppen im Hinblick auf ein geringes Niveau politischer Partizipation erhalten.
\end{abstract}

\section{Einleitung}

Eine wesentliche politische Intention hinter den Bildungsreformen der 1960er Jahre war die Schaffung einer Gesellschaft der politisch mündigen Bürgerinnen und Bürger und damit die Förderung politischer Beteiligung als Grundlage für eine funktionierende Demokratie. Damals wie heute ist eine breite politische Partizipation ein Qualitätsmerkmal einer Demokratie, entsprechend dem Grundsatz „citizen participation is at the heart of democracy“ (Verba et al. 1995: 129). ${ }^{1}$ Diese Ansicht vertrat im Vorfeld der Bildungsreformen vor allem Dahrendorf (1965), der „Bildung als Bürgerrecht“ einforderte, da er in einer steigenden Bildungsbeteiligung eine Chance sah, durch Höherbildung breiter Bevölkerungsschichten bessere Partizipations- und Gestaltungsmöglichkeiten, insbesondere bezüglich des politischen Systems zu eröffnen. Ein höheres Bildungsniveau sollte die Mündigkeit der Bürger fördern und letztlich die Demokratie stärken. Ziel war eine Demokratie, in der die Bürgerinnen und Bürger Politik nicht nur verstehen, sondern als rationale Akteure selbst gestalten sollten (vgl. auch Müller

* Wir danken Klaus Armingeon, Achim Goerres, Anna Hecken und Harald Schoen für wertvolle Hinweise und Anregungen.

1 So sieht Fuchs (2000) die Möglichkeiten politischer Beteiligung und die Annahme dieser Möglichkeiten durch die Bevölkerung als ein Qualitätsmerkmal der Demokratie an.

Kölner Zeitschrift für Soziologie und Sozialpsychologie, Jg. 59, Heft 3, 2007, S. 410-439. 
1998). Gefragt werden soll daher, ob im Zuge der Bildungsexpansion eine politische Mobilisierung stattgefunden hat, die eine Folge dieser Entwicklung hin zu einer höheren Bildungsbeteiligung ist. Sind die Entwicklungen von Bildungsexpansion und politischer Partizipation verbunden, und wenn ja: über welche Mechanismen?

Im Hinblick auf die nun schon länger andauernde Diskussion um „Politikverdrossenheit" - insbesondere der jungen Generationen -, die sich nach Meinung vieler Politiker und Journalisten in einer verstärkten Ablehnung von Politik und politischem Handeln zeigt, scheint schnell eine Antwort parat: Desinteresse statt politischer Mobilisierung. Dem allgemeinen Lamentieren über eine Abkehr von der Politik ist allerdings mit großer Skepsis zu begegnen (Lösche 1995). So ist eine Parteienverdrossenheit von einer generellen Politikverdrossenheit ${ }^{2}$ zu unterscheiden. Ebenso ist eine Auswirkung von Parteienverdrossenheit auf der Einstellungsebene auf das politische Handeln, insbesondere die Wahlbeteiligung, nicht belegt. Auch helfen zu einfache sozialwissenschaftliche Analysen aus einer querschnittlichen Perspektive an dieser Stelle nicht weiter, da sie häufig problematische Ergebnisse produzieren (Becker 2002). Stattdessen sind Längsschnittdaten nötig, die - soweit möglich - unter Berücksichtigung verschiedener Zeitdimensionen (Alter, Periode, Kohorte) zu analysieren sind. Dies ist unter anderem deshalb notwendig, weil die Bildungsexpansion einen Prozess in der Zeit darstellt, der entsprechender Untersuchungsmethoden bedarf.

Nachdem anhand einer solchen Untersuchungsstrategie unter simultaner Berücksichtigung von Alters-, Perioden- und Kohorteneffekten bereits gezeigt werden konnte, dass - im Gegensatz zur Wahrnehmung, das politische Interesse jüngerer Generationen sei gesunken - jüngere bzw. später geborene Kohorten unter Kontrolle ihres Alters und von Periodenmerkmalen tendenziell stärker politisch interessiert sind als ältere, früher geborene Kohorten (Hadjar und Becker 2006a), soll im Rahmen dieses Beitrags folgende Fragestellung bearbeitet werden: Wie hat sich unkonventionelle politische Partizipation - und damit ein politischer Gegenstand auf der Handlungsebene - über die Bildungsexpansion entwickelt? Lässt sich eine Verbindung zwischen Bildung und (unkonventioneller) politischer Partizipation auf der Individualebene herstellen? Und: Wurde eine entsprechende Entwicklung der (unkonventionellen) politischen Partizipation im Zeitverlauf auf der Aggregatebene von der Bildungsexpansion mit geprägt? Ziel dieses Beitrags ist somit sowohl eine Untersuchung zur Bildungsabhängigkeit politischer Partizipation unter Berücksichtigung ausgewählter sozialer Mechanismen (z.B. politische Wirksamkeitsüberzeugungen, politisches Interesse), die hinter der Beziehung zwischen Bildung und Partizipation stehen, als auch eine Analyse der Entwicklung unkonventioneller politischer Partizipation im Zeitverlauf. Dabei werden bildungsbezogene Aspekte fokussiert. ${ }^{3}$

2 Auch hier ist darauf hinzuweisen, dass die Verwendungen und die Bedeutungszuweisungen im Hinblick auf den Begriff der Politikverdrossenheit sowohl in der Öffentlichkeit als auch im sozialwissenschaftlichen Diskurs sehr vielfältig sind (vgl. Arzheimer 2002). Statt einer systematischen Definition scheint nur ein kleinster gemeinsamer Nenner erkennbar zu sein - eine gewisse Distanz zu Politik als wesentlicher Aspekt des Begriffs.

3 Entsprechend werden einige Variablen zur Reduktion der Komplexität der Untersuchung ausgeklammert. Dazu gehören u.a. politische Ziele, Rechts-Links-Einordnung und Zufriedenheit mit System, Demokratie und Regierung (vgl. Lengfeld et al. 2000) sowie Aspekte des Wertewandels. Wenngleich Befunde darauf hinweisen, dass der Wertewandel hin zum Postmaterialis- 
Im nächsten Abschnitt werden Hypothesen zum Zusammenhang zwischen Bildung und Partizipation auf der individuellen Ebene und damit in Beziehung stehende Hintergrundfaktoren sowie zur Entwicklung politischer Partizipation im Zeitverlauf theoretisch hergeleitet (Abschnitt II). Auf die Darstellung des Datenmaterials und der Messinstrumente, die in den jeweiligen Analysen verwendet wurden (Abschnitt III), folgen zwei empirische Untersuchungen (Abschnitt IV): Zunächst werden im Rahmen einer Querschnittsanalyse die Bestimmungsfaktoren unkonventioneller und konventioneller Partizipation untersucht, um schließlich unkonventionelle Partizipation im Zeitverlauf unter Berücksichtigung von Alters-, Perioden- und Kohorteneffekten hinsichtlich der Frage zu analysieren, inwieweit die Bildungsexpansion diese Entwicklung beeinflusst hat. In einem letzten Teil werden die Befunde zusammengefasst und diskutiert (Abschnitt V).

\section{Bildungsexpansion und unkonventionelle politische Partizipation}

Der Wandel der politischen Beteiligung im Zuge der Bildungsexpansion der letzten Jahrzehnte wird vor allem im Hinblick auf neue, unkonventionelle Formen politischer Partizipation thematisiert (Inglehart 1997), die besonders im Rahmen der "Studentenbewegung“ 1968 etabliert wurden. Diese sind ein Ausdruck eines „zunehmenden Verlangens nach Partizipation als Folge der Bildungsexpansion" (Kornelius und Roth 2004: 24), das hier primär mit zunehmenden Kompetenzen erklärt werden soll, aber sicher auch auf einen Wertewandel zurückgeht. Politische Partizipation ist zunächst allgemein als "freiwillige Handlungen mit dem Ziel der Beeinflussung politischer Entscheidungen" (Niedermayer 2001: 131; vgl. Barnes et al. 1979: 42; Verba et al. 1995: 38) zu fassen. Als unkonventionelle politische Partizipation werden jene Formen politischer Beteiligung verstanden, die, im Sinne unverfasster bzw. nicht auf verfasste Aktivitäten bezogener Beteiligungsformen, spontan oder geplant in relativer Distanz zu parteibezogenen politischen Institutionen entstehen (Kaase 2000). ${ }^{4}$ Zum Handlungsreper-

mus ebenfalls eine Folge der Bildungsexpansion ist (Hadjar 2006) und auch seinen Anteil an der Zunahme politischer Partizipation hat (Hadjar und Becker 2006b), würde eine detaillierte Betrachtung hier zu weit führen - zumal die Frage des Einflusses von Werthaltungen dringend komplexer Analysen bezüglich der Kausalität zwischen Werten und Verhalten bedarf. So wäre zu betrachten, inwieweit Werte über die Einstellungen bzw. als Rahmen für die Situationsdefinition das Handeln von Menschen beeinflussen und inwieweit die Handlungen von Menschen wiederum die in Befragungen von Individuen berichteten Werthaltungen - im Sinne nachträglicher Rationalisierungen - prägen. Bezüge zum Wertewandel werden daher nur in der Diskussion zur Interpretation der Befunde unter Rückgriff auf Erkenntnisse anderer empirischer Studien hergestellt.

4 Eine adäquate Begrifflichkeit für die hier fokussierten Formen sogenannter „unkonventioneller" Partizipation zu finden ist schwer. Im Hinblick auf den im Folgenden verwendeten Begriff der unkonventionellen Partizipation ist zu problematisieren, dass darunter auch Verhaltensweisen gefasst werden, die sich über die Zeit etabliert haben und von wachsenden Teilen der Bevölkerung akzeptiert und genutzt werden (vgl. Niedermayer 2001). So ist zu fragen, ob eine Form der Beteiligung, wie die Teilnahme an einer Demonstration, heute noch unkonventionell ist. Welche Beteiligungsformen unter den Begriff „unkonventionell“ fallen, ist somit einer zeitlichen Entwicklung unterworfen. Wenn nun von unkonventioneller Partizipation gesprochen wird, bezieht sich dies auf die Beteiligungsformen, die vor der "partizipatorischen Revolution“ 
toir unkonventioneller Partizipation gehören nach der Political Action Study (Barnes et al. 1979) das Schreiben von Parolen an Wände, die Beteiligung an Unterschriftensammlungen, die Beteiligung an Boykotts, die Teilnahme an genehmigten politischen Demonstrationen, die Weigerung der Zahlung von Mieten, Raten oder Steuern, die Beteiligung an wilden Streiks, Besetzungen von Fabriken, Ämtern und anderen Gebäuden, das Aufhalten des Verkehrs durch eine Demonstration sowie die Beschädigung fremden Eigentums und die Anwendung von Gewalt gegen Personen.

Aus der Taxonomie von Uehlinger (1988: 110) lassen sich konventionelle und unkonventionelle Formen politischer Partizipation folgendermaßen gegeneinander abgrenzen: Der konventionellen Partizipation zuzuordnen sind parteiorientierte Beteiligungsformen (Parteiarbeit, politische Gruppierung, Wahlhelfer, politisches Amt). Problemspezifische Aktivitäten (Bürgerinitiative, Unterschriftensammlung, Briefe an Zeitung, genehmigte Demonstrationen etc.) und ziviler Ungehorsam (verbotene Demonstrationen, Konfrontation mit der Polizei, Hausbesetzungen etc.) gehören demgegenüber zu den unkonventionellen Partizipationsformen. ${ }^{5}$ Unkonventionelle Formen sind somit stärker protestorientiert, weniger institutionalisiert, außerhalb des Rahmens verfasster Parteienpolitik und verstärkt demonstrativ, provokativ und herausfordernd (vgl. Lengfeld et al. 2000). Das Kriterium der Legalität oder Normkonformität, wie es etwa Opp (1985) thematisiert, eignet sich nur sehr eingeschränkt zur Abgrenzung zwischen konventionellen und unkonventionellen Partizipationsformen, da empirische Studien für einige Formen unkonventioneller Beteiligung (z.B. Unterschriftensammlungen, Demonstrationen) einen großen Rückhalt in der Bevölkerung belegen und zum anderen die Definition, was den Normen entspricht, starken zeitlichen Veränderungen ausgesetzt ist (vgl. Uehlinger 1988: 29). Formen unkonventioneller Partizipation können somit legal und illegal sein, wie dies in der Taxonomie von Uehlinger (1988) bereits thematisiert wurde.

Dabei schließen sich unkonventionelle und konventionelle Partizipationsformen nicht gegenseitig aus, sondern ergänzen sich. So zeigen empirische Befunde, dass konventionelle und unkonventionelle Formen politischen Handelns miteinander korrelieren (vgl. Opp 1985: 282). Entsprechend der These von Kaase (1984; van Deth 1997)

(Kaase 1984) in den 1960er Jahren als unkonventionell galten. Eine andere mögliche Unterscheidung in institutionalisierte und nicht-institutionalisierte Formen politischer Beteiligung erscheint als weniger sinnvoll: Unkonventionelle Beteiligungsformen sind zu einem geringeren Grad oder teilweise gar nicht institutionalisiert. Es ist dennoch nicht plausibel, dass sie, wie Kornelius und Roth (2004: 27) dies beschreiben, per definitionem „ohne institutionellen Rahmen" stattfinden. Zu nennen sind hier gesetzliche Rahmen, welche die Grenzen legaler unkonventioneller Partizipation festschreiben, andererseits aber auch institutionelle Strukturen (z.B. von Bürgerinitiativen), in deren Rahmen solche Partizipationsformen ausgeübt werden. Letztlich stellen die unkonventionellen politischen Handlungsmuster im soziologischen Sinne selbst Institutionen im Sinne von Verhaltensregelmäßigkeiten dar.

5 Einen Sonderstatus jenseits der Dimensionen konventioneller und unkonventioneller Partizipation nehmen nach Kaase (2000; Barnes et al. 1979) Aktivitäten ein, die sich aus der Staatsbürgerrolle ergeben (Ausübung des Wahlrechts, Meinung sagen) sowie politische Gewalttaten gegen Personen oder Sachen. Erstere Formen wären eigentlich der konventionellen Partizipation zuzuordnen, letztere der unkonventionellen Partizipation; ein Problem bei der empirischen Zuteilung zu den Dimensionen ergibt sich jedoch durch die einerseits sehr hohe Auftretenswahrscheinlichkeit (Wahlbeteiligung) sowie die andererseits im Vergleich sehr niedrige Auftretenswahrscheinlichkeit (Gewalttaten). 
zur "partizipatorischen Revolution“ hat sich in den 1960er Jahren das politische Beteiligungsverhalten der Bevölkerung dahingehend gewandelt, dass zusätzlich zu den konventionellen Partizipationsformen (Wählen, Parteimitarbeit etc.) neue Formen politischen Protests hinzugekommen sind. Vor allem legale Formen unkonventioneller politischer Partizipation wurden zunehmend von einem Großteil der Bevölkerung anerkannt (Niedermayer 2001: 215). Auch neueste Studien belegen, dass unkonventionelle Partizipationsformen weiterhin „tendenziell [...] mehr als Ergänzung denn als Alternative genutzt" werden (Kornelius und Roth 2004: 14). Bezüglich konventioneller Beteiligung sind inaktive Individuen dies auch im Hinblick auf unkonventionelle Beteiligung.

1. Bildung, Status, Political Efficacy, politisches Interesse und unkonventionelle politische Partizipation

Ein starker Zusammenhang zwischen Bildungsniveau und politischer Aktivität gehört zu den Standardbefunden der Partizipationsforschung (vgl. Uehlinger 1988; Leighley 1995). Die formale Bildung ist einer der wichtigsten Bestimmungsfaktoren - und dies sowohl im Hinblick auf konventionelle, als auch auf unkonventionelle Beteiligungsformen: Individuen mit höherer Bildung sind aktiver bzw. beteiligen sich stärker politisch als niedriger Gebildete. Darauf weisen bereits Befunde aus klassischen Partizipationsstudien (Kaase und Marsh 1979; Milbrath und Goel 1977) hin. Im Hinblick auf die Typisierung von Uehlinger (1988: 171) zeigt sich, dass sowohl der Anteil der Parteiaktiven, als auch der Anteil der gewaltlosen Aktivisten mit zunehmendem Bildungsniveau steigt, wobei vor allem für den zivilen Ungehorsam als Kernaspekt unkonventioneller Partizipation ein positiver Bildungseffekt zu konstatieren ist.

Bei der theoretischen Exploration einer Beziehung zwischen Bildungsniveau und unkonventioneller politischer Partizipation sind bestimmte Mediatoren zwischen diesen beiden Faktoren in Betracht zu ziehen. Zunächst sollen zwei Sichtweisen auf die Rolle des Bildungsniveaus thematisiert werden (vgl. Hadjar 2006: 206-208). Zum einen ist Bildung als Humanvermögen zu fassen, d.h. als kognitive Fähigkeiten, Reflexions- und Handlungskompetenzen. Eine höhere Bildung bedeutet damit einen erleichterten $\mathrm{Zu}$ gang zu Politik (Krimmel 2000: 628), denn es sinken die Kosten, sich mit Politik zu beschäftigen und z.B. politische Einstellungen zu reflektieren. ${ }^{6}$ So existieren u.a. empirische Belege dafür, dass höher gebildete Schichten verstärkt nach situativen Einschätzungen sozialer und politischer Bedingungen reflektiert wählen, statt einer Partei habituell zuzuneigen (Dalton 1984). Zum anderen stellt Bildung aber auch ein Humankapital dar, d.h. Bildung in Form von akkumulierten Bildungszertifikaten lässt sich im späteren Erwerbsleben in Arbeitsmarktpositionen und Einkommen umsetzen (Becker 1974) und bestimmt so die spätere soziale Lage und die Lebenschancen (Becker 1998). Diese Deutung von Bildung als Humankapital basiert auf dem Statuszuweisungskonzept von Mayer und Blossfeld (1990), nach dem die soziale Herkunft (sozialer Status

6 „Education helps citizens to understand and evaluate politics and political options, facilitates deliberation, clarifies the importance of a working democracy for their own lives, and helps them handle frustrations in the domain of politics" (Armingeon 2007: 362). 
der Eltern) den erreichten Schulabschluss bestimmt; der Schulabschluss wiederum hat einen substantiellen Anteil an der Erklärung des späteren Berufsstatus. ${ }^{7}$ Es kann somit ein Effekt der Bildung über die soziale Lage auf politische Einstellungen und Verhalten vermutet werden. Armingeon (2007) fokussiert bezüglich des Effekts auf politische Partizipation vor allem das höhere Einkommen, das aus einem höheren Bildungsabschluss resultiert und das für konventionelle und unkonventionelle politische Unternehmungen herangezogen werden kann.

Ins Zentrum der Exploration der Beziehung zwischen Bildung und politischer Partizipation ist zunächst der Faktor der „Political Efficacy“ zu stellen. Ein höheres Bildungsniveau geht mit der Wahrnehmung einer höheren politischen Selbstwirksamkeit (Vetter 2000; Becker 2004) einher. Political Efficacy stellt eine Kontroll- bzw. Wirksamkeitsüberzeugung bezüglich politischen Handelns dar - „the feeling that individual political action does have, or can have, an impact upon the political process" (Campbell et al. 1954: 187). Diese hat zwei Dimensionen: Während sich die internale politische Wirksamkeitsüberzeugung auf die Wahrnehmung, dass Instrumente zur politischen Teilhabe individuell zur Verfügung stehen, bezieht, manifestiert sich in der externalen politischen Wirksamkeitsüberzeugung die Wahrnehmung, dass Autoritäten und Regimes auf Einflussversuche über politische Partizipation auch reagieren (vgl. Vetter 2000). Die These ist nun, dass ein höheres Bildungsniveau zu einer höheren internalen und externalen Political Efficiacy führt (vgl. Abramson 1983), weil höhere Bildung einerseits bessere Reflexionsfähigkeiten und Kompetenzen sowie ein höheres politisches Wissen (Jennings 1996) bedeutet, und andererseits mit höherer Bildung bessere Aufstiegschancen und ein höherer sozialer Status einhergehen, der - zumindest in der subjektiven Wahrnehmung - höhere Kompetenzen bezüglich der Handlungsmöglichkeiten in der Gesellschaft und im politischen System verspricht (vgl. Krebs und Schmidt 1993: 100). Entsprechend postulierten und belegten bereits Milbrath und Goel (1977: 58) aus eher psychologischer Perspektive: „persons who feel efficacious participate at a higher level than those who lack such feelings". Auch Barnes et al. (1979) zeigen im Rahmen der Political Action Study, dass ein höherer sozialer Status mit einem stärkeren politischen Engagement verknüpft ist. Nach Befunden von Uehlinger (1988: 179), der Political Efficacy als subjektive politische Kompetenz bezeichnet, führt eine höhere politische Wirksamkeitsüberzeugung zu einer höheren Wahrscheinlichkeit, zu den Partizipationstypen der Parteiaktiven und den gewaltlosen, unkonventionellen Aktivisten zu gehören.

Die höhere politische Wirksamkeitsüberzeugung führt zu einem verstärkten politischen Interesse, d.h. zu einem höheren Grad der Neugier der Bevölkerung an Politik (van Deth 1990: 278) bzw. an Personen, Prozessen und Vorgängen in der Sphäre der Politik. Unter Rückgriff auf die rationale Perspektive (Becker 1974; vgl. Hadjar und Becker 2006a) lässt sich argumentieren, dass nur, wer die Erfolgswahrscheinlichkeit, durch politische Partizipation Wirkung zu erzielen und politisch denken und handeln zu können, als hoch einschätzt sowie wer aufgrund seiner Kompetenzen (Informationsverarbeitungsfähigkeiten, Wissen etc.) die Kosten für die Auseinandersetzung mit poli-

7 Diese Mechanismen der Statuszuweisung sind relativ persistent; das Modell hat auch über die Bildungsexpansion nichts an empirischer Geltung verloren (vgl. Becker 2003). 
tischen Themen als gering einschätzt, sich in besonderem Maße politisch interessieren wird.

Das politische Interesse bildet im Rahmen dieser Betrachtung das Bindeglied für die Beziehung zwischen Bildung, Status, Political Efficacy und politischer Partizipation. Das politische Interesse bezieht sich auf den Grad der Involviertheit in Politik bzw. den „degree to which citizens are interested in and concerned about politics and public affairs" (Milbrath und Goel 1977: 46). ${ }^{8}$ Als konative, d.h. als handlungs-, antizipationsund einflussbezogene Komponente des Einstellungssystems gilt es als Vorstufe für politisches Engagement (vgl. Breckler 1984; van Deth 1990), d.h. politisches Interesse bildet in vielen Fällen den Auslöser für einen „switch from passivity to participation “ (Armingeon 2007: 363). Ein Mangel an politischem Interesse reduziert die politische Partizipationsbereitschaft der Individuen (Milbrath und Goel 1977; Barnes et al. 1979). Einen Zusammenhang zwischen politischem Interesse und politischer Partizipation belegt auch Uehlinger (1988: 174): Mit steigendem politischen Interesse erhöhen sich die Anteile der Partizipationstypen „parteiorientierte Partizipation“ (konventionelle Beteiligungsformen) sowie „problemspezifische Partizipation“ und „ziviler Ungehorsam“ (unkonventionelle Beteiligungsformen). Somit ist nun auch für unkonventionelle politische Beteiligung zu vermuten: Aus einem höheren politischen Interesse folgt, durch Drittvariablen (u.a. Problemwahrnehmung, Zufriedenheit mit der Regierung; vgl. u.a. Kornelius und Roth 2004) moderiert, eine größere Wahrscheinlichkeit, sich im Rahmen unkonventioneller politischer Partizipation zu engagieren.

Aus den hier dargestellten Überlegungen soll als wesentliche Annahme folgende Hypothese (H1) formuliert werden:

H1: Mit steigendem Bildungsniveau steigt die Wahrscheinlichkeit unkonventioneller politischer Partizipation an.

\section{Bildungsexpansion und unkonventionelle politische Partizipation}

$\mathrm{Zu}$ den wesentlichen Folgen der Bildungsexpansion gehören zwei mit der zunehmenden Bildungsbeteiligung und Höherqualifikation einhergehende soziale Mechanismen: Während die „kognitive Mobilisierung" als erwartete und erwünschte Folge der Bildungsexpansion gelten kann, hat die ebenso im Zuge der Bildungsexpansion erwartete „Heterogenisierung" neben dem (leichten) Abbau von Bildungsungleichheiten auch unerwünschte Wirkungen in Gestalt sinkender Standards der höheren Bildungsinstitutionen und -schichten (vgl. Hadjar und Becker 2006b).

Die kognitive Mobilisierung als unmittelbare Folge der Bildungsexpansion führte zu einem langfristigen gesellschaftlichen Wandel und ist Ursache des kulturellen Um-

8 Dabei soll hier politisches Interesse weniger aus einem psychologischen Verständnis heraus als Persönlichkeitseigenschaft verstanden werden, sondern stattdessen, der soziologischen Perspektive folgend, als Aktivierungszustand, der durch situative Faktoren wie verfügbare Ressourcen (bezüglich der Partizipation) und die Situationsdefinition bzw. -wahrnehmung bestimmt wird (vgl. Schmidtchen und Uehlinger 1983: 188). Daher ist zu erwarten, dass politisches Interesse und Partizipationsbereitschaft "gleichermaßen nicht nur lebenszyklischen, sondern auch situationalen Effekten unterliegen" (Uehlinger 1988: 173). 
bruchs, der Ende der 1960er Jahre zuerst eine bestimmte Studentengeneration erfasste (Baumert 1991: 347; Baumert et al. 2003). Die kognitive Mobilisierung auf der Aggregatebene ist, entsprechend der Aggregationslogik, das Abbild der Beziehung zwischen individuellem Bildungsniveau und individuellen Fähigkeiten der Informationsverarbeitung im Umgang mit Problemstellungen bzw. Handlungskompetenzen. Auf der gesellschaftlichen Ebene vollzog sich im Zuge der Bildungsexpansion somit eine Entwicklung hin zu „verbesserte[n] Teilhabe- und Gestaltungsmöglichkeiten in vielen Bereichen des privaten und öffentlichen Lebens" (Baumert 1991: 347). Die kognitive Mobilisierung hat zu einer Erweiterung der Fähigkeiten im Umgang mit Politik bzw. politischer Handlungskompetenz und letztlich zu einer "political sophistication of Western mass publics" (Dalton 1984: 265; vgl. Inglehart 1977) geführt. Während diese Entwicklung zu den erwünschten Folgen der Bildungsreformen zählt (Dahrendorf 1965), zog die Heterogenisierung der Schülerschaft höherer Schulen wie auch die Homogenisierung der Schülerschaft der Hauptschule als niedrigster Schulform (Solga und Wagner 2001) unerwünschte Konsequenzen in Form tendenziell sinkender Standards nach sich. Die Öffnung der Aufstiegsschulzweige bzw. der höheren Bildungseinrichtungen für vormals exkludierte Schichten (z.B. Landwirte und Arbeiter) führte zu einer heterogeneren Zusammensetzung der Schülerschaft in höheren Schulformen; erkennbar an einem sich abschwächenden Zusammenhang zwischen sozialer Herkunftsschicht und Bildungsniveau (Becker 2003). Bereits Schelsky (1956) merkte an, dass eine Erweiterung der Bildungsmöglichkeiten und eine entsprechende soziale Heterogenisierung der Gruppe der höher Gebildeten nur unter Inkaufnahme sinkender Bildungsstandards bzw. eines sinkenden Niveaus in dieser Gruppe möglich sei. Somit sollten die höher Gebildeten hinsichtlich ihrer Fähigkeiten, Kompetenzen, Orientierungen und Verhaltensmuster eine zunehmend weniger distinkte Position gegenüber den geringer Gebildeten einnehmen (Baumert 1991). Alles in allem sollte die hier thematisierte Heterogenisierung der höheren Bildungsschicht die Effekte der Bildungsexpansion abschwächen, was eine geringere Niveauverschiebung in der Gesamtbevölkerung zur Folge hätte (vgl. Müller 1998). Im Hinblick auf die Entwicklung der unkonventionellen politischen Partizipation ist eine leichte Tendenz zu erwarten, dass sich die höhere Bildungsschicht in ihrem Ausmaß an unkonventioneller Partizipation über die Kohortenabfolge auf die niedrigen Bildungsschichten zubewegt, weil in die höhere Bildungsschicht nun nicht mehr nur Individuen aus Herkunftsfamilien mit höherer Bildung, einem hohen Sozialstatus (und damit auch einer höheren Kompetenzwahrnehmung bezüglich politischer Reflexion und politischen Handelns sowie höherem politischen Interesse) vordringen, sondern auch politikferne Individuen. Die negative Distinktion der am niedrigsten Gebildeten sollte in Anbetracht der Homogenisierung der Schülerschaft von Hauptschulen als „Restschule“ (Solga und Wagner 2001) erhalten bleiben bzw. sogar zunehmen.

3. Unkonventionelle politische Partizipation im Zeitverlauf

Bei der Analyse sozialer Entwicklungen reicht die Betrachtung einzelner zeitlicher Effekte nicht aus, denn diese Vorgehensweise kann zu „temporalen Fehlschlüssen“ führen. 
Als temporale Fehlschlüsse sollen hier falsche Schlussfolgerungen, die auf der Analyse nur eines temporalen Effekts basieren, verstanden werden. Stattdessen ist zu beachten, dass es drei temporale Dimensionen gibt, die im Hinblick auf sozialen Wandel Berücksichtigung finden sollten: Alters-, Perioden- und Kohorteneffekte (A-P-K-Analyse; Mayer und Huinink 1990; Bürklin et al. 1994; Hadjar und Becker 2006a). Das Separieren dieser drei temporalen Effekte dient der besseren Erklärung der hinter dem Wandel liegenden Kausalmechanismen. Es ist zu fragen, ob und inwieweit die unterschiedlichen Sozialisationserfahrungen politischer Generationen, Alterungsprozesse bzw. unterschiedliche Positionen im Lebenszyklus oder periodenspezifische Zustände und Ereignisse ausschlaggebend für Veränderungen im politischen Partizipationsverhalten sind. Die Sinnhaftigkeit einer solchen Herangehensweise zeigt sich u.a. in Befunden zum Wandel des politischen Interesses oder zum Wertewandel: Während bei Betrachtung von Geburtskohorten das politische Interesse der zwischen 1959 und 1968 Geborenen geringer zu sein scheint als das politische Interesse früherer Generationen, zeigt sich im komplexen A-P-K-Modell, dass die jüngere Generation, unter Kontrolle ihres geringeren Alters sowie Periodeneinflüssen, sogar ein ausgeprägteres politisches Interesse hat (Hadjar und Becker 2006a).9 Entsprechend soll nun auch die Entwicklung der unkonventionellen politischen Partizipation als Kompositionseffekt, d.h. als Zusammenspiel sich gegenseitig überlagernder Effekte der historischen Lagerung und Prägung von Kohorten durch gesellschaftliche Verhältnisse, der individuellen Entwicklung im Lebenszyklus sowie der historischen Perioden, thematisiert werden (vgl. u.a. Becker und Mays 2003).

Vor dem Hintergrund der Ausführungen zum Zusammenhang zwischen Bildung und politischer Partizipation ist ein Kohorteneffekt anzunehmen, in dem die zunehmende "kognitive Mobilisierung" in der Abfolge von Geburtsjahrgängen (Baumert et al. 2003) zum Ausdruck kommt, die zu besseren politischen Kompetenzen, höherem politischen Interesse und verstärkter politischer Beteiligung geführt hat. Diese These geht konform mit den theoretischen Überlegungen und Befunden zur „partizipatorischen Revolution" (Kaase 1984), deren Träger höher gebildete, jüngere Generationen sind, die vor allem unkonventionelle politische Partizipationsformen neben den klassischen konventionellen Formen gesellschaftsfähig machen. Früher geborene Kohorten sollten demnach im geringeren Ausmaß unkonventionell partizipieren als später geborene Kohorten. ${ }^{10}$

9 Diekmann (2004: 283-287) zeigt zwei Beispiele solcher Fehlschlüsse auf: Als Lebenszyklusfehlschluss wird der Befund thematisiert, dass mit zunehmendem Alter die Intelligenz abnimmt, denn bei gleichzeitiger Betrachtung von Kohortenunterschieden erweisen sich jüngere Kohorten zwar als intelligenter, der Grad an Intelligenz bleibt aber über den Lebenslauf bzw. das Alter stabil. Als idealtypisches Beispiel für einen Kohortenfehlschluss gilt die Vorstellung zum Wertewandel, wonach das Sinken des Materialistenanteils ein reiner Kohorten- bzw. Generationeneffekt ist (Inglehart 1977). Stattdessen zeigt sich in der simultanen Analyse von Alter und Kohorte, dass zumindest teilweise ein Alterseffekt hinter diesen Ergebnissen steht, da jüngere Individuen im geringeren Ausmaß materialistischen Werten anhängen (vgl. Hadjar 2006).

10 Ein Kohorteneffekt für politische Partizipation erscheint ebenso aus Sicht von Inglehart (1997) als plausibel, der von einer Zunahme politischer Partizipation - und hier: vor allem unkonventioneller Beteiligungsformen - über die Kohortenabfolge ausgeht, die bedingt ist durch einen 
Entsprechend dem Verlauf der Bildungsexpansion müsste ein wesentlicher Anstieg der politischen Partizipation erstmals für die Kohorte der 1949 bis 1958 Geborenen zu konstatieren sein (vgl. Meulemann 1987), die 1959-68er Geburtsjahrgänge sollten dann bei alleiniger Betrachtung der Kohorten die höchsten Werte aufweisen. Die zunehmende Heterogenität höherer Bildungsschichten sollte den Bildungs- und den Kohorteneffekt in seiner Stärke bzw. seiner statistischen Signifikanz abschwächen. Bei Interpretation der Kohorten im Hinblick auf das Bildungsniveau ergeben sich aus den beiden Argumentationen zur kognitiven Mobilisierung (vgl. Baumert 1991; Dalton 1984) und zur Heterogenisierung der höheren Bildungsgruppe (Schelsky 1956; Baumert 1991) bzw. zur Homogenisierung der niedrig Gebildeten (Solga und Wagner 2001) folgende Hypothesen (H2a, H2b):

H2a: Jüngere Kohorten zeigen infolge ihrer höheren Bildungsbeteiligung eine stärkere unkonventionelle politische Partizipation.

H2b: Der Unterschied in der unkonventionellen politischen Partizipation zwischen höherer und mittlerer Bildungsgruppe ist in den jüngeren Kohorten geringer ausgeprägt, während die niedrigste Bildungsgruppe ihre negative Distinktion beibehält.

Im Sinne der A-P-K-Analyse werden die Kohorteneffekte unter simultaner Berücksichtigung von Perioden- und Alterseffekten betrachtet, die in den multivariaten Modellen kontrolliert werden. Es ist von einer Überlagerung der Kohorteneffekte durch einen Lebenszyklus- bzw. Alterseffekt auszugehen. So kann, Uehlinger (1988: 173) folgend, aus der Situations- und Ressourcenbestimmtheit politischen Interesses und politischer Partizipation auf lebenszyklische Effekte geschlossen werden. Die Frage nach der Richtung dieses Alters- bzw. Lebenszykluseffekts erscheint als diffizil: Bezüglich der generellen politischen Partizipation ist zunächst von einer mit dem Alter ansteigenden Partizipation auszugehen. Nach einem Höhepunkt im mittleren Alter sollte das Ausmaß politischer Partizipation ca. ab dem 60. Lebensjahr tendenziell nachlassen (vgl. Milbrath und Goel 1977: 114). Hintergrundannahme ist, dass mit steigendem Alter zum einen politische Orientierungen sowie politische Handlungskompetenzen im Zuge der politischen Sozialisation sich erst entwickeln (Hoffmann-Lange 1993; Bürklin et al. 1994) und zudem mit dem Alter, d.h. der längeren Lebenszeit, auch die Opportunitäten steigen, sich politisch zu engagieren. Ein sehr junger Mensch dürfte nur wenige Gelegenheiten zu politischer Partizipation gehabt haben (vgl. Becker und Mays 2003). So formuliert Armingeon (2007: 362) im Hinblick auf die soziale Integration und Sozialisationsumwelten als Bestimmungsfaktoren für politische Beteiligung: „After adolescence the integration into family life as well as working life becomes stronger and the likelihood of being drawn into politics increases. Paying taxes as well as enjoying the benefits of the welfare state provide lessons about politics and demonstrate its relevance; and family members or workplace colleagues may provide a stimulus or social pressure

Wertewandel hin zu Partizipationswerten. Auch Herz (1987: 58) nimmt an, dass „Werte, die mit gesellschaftlich-politischer Partizipation " zu tun haben, eine historische Verankerung besitzen und sich nach dem Modell des Generationenwechsels verändern. Der Aspekt des Wertewandels soll aus Gründen der Reduktion des Untersuchungsrahmens hier nicht näher erörtert werden. 
towards increased political participation". Empirische Evidenzen für entsprechende Alterseffekte finden sich u.a. in Bezug auf konventionelle Partizipation wie Parteiaktivität, Wahlverhalten und Diskussionen (Uehlinger 1988: 168) sowie auf politisches Interesse (Hadjar und Becker 2006a). Nach der Beendigung des Arbeitslebens ist mit einer Stagnation des Anstiegs in der Partizipation oder sogar einer tendenziell zurückgehenden Partizipation zu rechnen, da dann entsprechende Sozialisationsumwelten und Anlässe nur noch im geringeren Maße gegeben sind (Armingeon 2007). Diese theoretischen Überlegungen und empirischen Befunde könnten zunächst im Rahmen einer Hypothese (H3) auf unkonventionelle Partizipation bezogen werden:

H3: Mit zunehmendem Alter steigt zunächst die Wahrscheinlichkeit unkonventioneller politischer Partizipation an, um im späteren Lebensverlauf (um das 60. Lebensjahr) zu stagnieren.

Unter besonderer Berücksichtigung des Wesens unkonventioneller politischer Partizipation bietet sich aber auch eine andere Argumentation an. Nach Befunden der Political Action Study (Barnes et al. 1979) sowie von Uehlinger (1988: 168) ist unkonventionelle politische Partizipation eher in jüngeren Altersgruppen zu finden. So finden sich Protestierer und gewaltlose Aktivisten im vergleichsweise höchsten Ausmaß in der Altersgruppe der 16- bis 25-Jährigen. Problemzentrierte Partizipation, die ebenfalls unkonventionellen Beteiligungsformen zuzuordnen ist, hat ihren Höhepunkt zwischen dem 26. und 35. Lebensjahr (Uehlinger 1988: 168). ${ }^{11}$ Als Erklärungsmuster für einen solchen Zusammenhang kämen einerseits ressourcentheoretische Erklärungen in Frage: Die größere Protest-Wahrscheinlichkeit junger Menschen wird von Barnes et al. (1979: 101) damit begründet, dass Jugendliche relativ frei von alltäglichen Verantwortlichkeiten für Karriere und Familie sind und mehr Zeit für unkonventionelle politische Aktivitäten haben. Zudem können jüngere Altersgruppen bei Protest- bzw. unkonventionellem Partizipationshandeln auf eine höhere physische Vitalität zurückgreifen. Aus entwicklungspsychologischer Perspektive ließe sich nach Kohlberg (1996) vermuten, dass die Heranziehung tendenziell risikoreicher, weniger legaler und normativ akzeptierter Beteiligungsformen auf im Jugendalter noch existierende Defizite an moralischen Kompetenzen zurückzuführen sei. In eine ähnliche Richtung, wenngleich stärker auf politische Kompetenzen bezogen, geht die Argumentation von Schmidtchen und Uehlinger (1983) bzw. Uehlinger (1988: 168f.): „Kommt noch bescheiden ausgebildete politische Kompetenz mit hohen Anforderungen in Form psychologisch schwer zu bewältigenden politischen Nachrichten zusammen, so kann daraus Angst und daraus Abwehr in Form von Aggression resultieren", wobei hier Aggression sicherlich nicht nur im engen Sinne als Vorform von Gewalt zu verstehen ist, sondern eher im weiteren Sinne als eine verstärkte Motivation, Einfluss zu üben. ${ }^{12}$

11 Diese Befunde gelten interessanterweise in den meisten Aspekten sowohl bezüglich aktueller politischer Aktivität, als auch im Hinblick auf bisherige politische Partizipation, d.h. akkumulierter Beteiligung (Uehlinger 1988: 169).

12 Andererseits könnte auch aus der Werteforschung ein negativer Alterseffekt abgeleitet werden: Eine mit dem Alter abnehmende unkonventionelle Aktivität wäre dann auf eine lebenszyklusbedingte Abnahme Inglehartscher postmaterialistischer Werte bzw. eine Zunahme an Pflichtund Akzeptanzwerten nach Klages zurückzuführen, die mit einer Abwendung von unkonventionellen Partizipationsformen verbunden sind. So zeigen verschiedene Studien, dass Kohor- 
An der Hypothese H3 zum Alters- bzw. Lebenszykluseffekt soll trotz dieser möglichen konträren Sichtweise zunächst festgehalten werden, da zum einen angenommen werden kann, dass sich im Zuge der partizipatorischen Revolution (Kaase 1984) Formen der unkonventionellen politischen Partizipation in der Gesamtbevölkerung, und damit auch in älteren Altersgruppen, etablieren konnten (vgl. Uehlinger 1988: 29; Niedermayer 2001: 215), und sich andererseits die abhängige Variable auf bisherige unkonventionelle Partizipation und nicht auf aktuelles Beteiligungsverhalten bezieht. Dieser Aspekt der Akkumulation würde vermuten lassen, dass mit jedem Lebensjahr die Chance zur Akkumulation von Partizipationsverhalten steigt, wobei ab dem Pensionsalter mit einer Stagnation zu rechnen wäre.

Empirische Befunde weisen zudem darauf hin, dass das Ausmaß politischer Partizipation von Periodeneffekten, also Wirkungen gesellschaftlicher Verhältnisse und politischer Ereignisse zu den jeweiligen historischen Kalenderzeiten, abhängig ist. Besonders bei gesellschaftlichen Problemlagen von hoher Salienz und einer hohen Ungerechtigkeitswahrnehmung ist mit verstärkter, vor allem auch unkonventioneller, politischer Partizipation zu rechnen (Lengfeld et al. 2000). Entsprechend sieht Klages (1984) politisches Interesse und politische Partizipation als Ausdruck der Unzufriedenheit mit dem Regierungs- bzw. Politikerhandeln. Mit einer zunehmenden Problemwahrnehmung, etwa bezüglich der Arbeitslosigkeit, sinkt das „unpolitische Staatsvertrauen“ und damit wachsen das politische Interesse und die Wahrscheinlichkeit (vor allem unkonventioneller) politischer Beteiligung. Eine Betrachtung des Erhebungszeitraums zwischen 1988 und 2000 weist auf mehrere politische bzw. politisch relevante Ereignisse hin, die eine temporäre verstärkte politische Beteiligung erwarten lassen. Sie wäre für die Zeit um 1988 und 1990 (hohe Arbeitslosigkeit, Vereinigung beider deutscher Staaten), um 1998 (hohe Arbeitslosigkeit, „Burn-out“ der 16-jährigen christlich-liberalen Regierungskoalition) oder die Zeit nach 1998 (Aufbruch, die ersten Jahre der rot-grünen Ära) ein Anstieg politischer Partizipation zu erwarten (vgl. Hadjar und Becker 2006a). $\mathrm{Da}$ diese verschiedenen Periodeneinflüsse kaum als linearer Trend angenommen werden können, wird folgende ungerichtete Hypothese (H4) aufgestellt:

H4: Bildungs- und Kohorteneffekte werden von Periodeneffekten, die auf gesellschaftlichen Ereignissen und sozialstrukturellen Veränderungen basieren, überlagert.

Im Rahmen der Längsschnittanalysen zur Entwicklung unkonventioneller politischer Partizipation wird zudem das Geschlecht kontrolliert. Ein Grund dafür ist zunächst, dass empirische Befunde für die Geschlechtsspezifizität politischer Partizipation sprechen. Frauen haben auch nach der partizipatorischen Revolution noch ein geringeres politisches Interesse und fühlen sich weniger politisch kompetent als Männer, wobei die Geschlechterunterschiede in der unkonventionellen Partizipation geringer sind (Westle 2001). Des Weiteren soll damit berücksichtigt werden, dass Frauen insbesondere von der Bildungsexpansion profitiert haben. Im Zuge der Bildungsexpansion hat vor allem ein Anstieg der Bildungsbeteiligung von Frauen und damit ein Abbau geschlechtsspezifischer Ungleichheiten stattgefunden, während etwa Bildungsungleichhei-

teneffekte auf den individuellen Wertekosmos durch Alterseffekte, die entgegengesetzt gerichtet sind, überlagert werden (vgl. Hadjar 2006). 
ten nach sozialer Herkunft nur im geringeren Maße abgenommen haben (vgl. Hecken 2006). Schließlich ist die Berücksichtigung des Geschlechts auch deshalb sinnvoll, weil Frauen durch ihre höhere Lebenserwartung (Klein et al. 2006) besonders in den höheren Altersgruppen in größerer Anzahl in den kumulierten Längsschnitt eingehen.

\section{Untersuchungsdesign, Datensatz und Messinstrumente}

Da nicht alle interessierenden Variablen über längere Zeiträume erhoben wurden, muss die Untersuchung der Fragestellungen auf zwei Teilanalysen aufgeteilt werden. Während die sozialen Mechanismen zwischen Bildungsniveau und politischer Partizipation im Rahmen eines Strukturgleichungsmodells analysiert werden, wird die unkonventionelle politische Partizipation im Zeitverlauf anhand einer A-P-K-Analyse untersucht.

\section{Strukturgleichungsmodell zur Erklärung politischer Partizipation}

Zunächst wird zur Exploration sozialer Hintergrundmechanismen des Zusammenhangs zwischen Bildung und Partizipation ein Strukturgleichungsmodell zur Erklärung konventioneller und unkonventioneller politischer Beteiligung geschätzt. Die Schätzung erfolgt nach der Maximum-Likelihood-Methode und basiert auf einer Korrelationsmatrix unter ausschließlicher Nutzung manifester Variablen mittels AMOS.

a) Datenbasis: Die Datenbasis bildet ein Datensatz, in dem die ALLBUS-Erhebungen 1988 und 1998 gepoolt wurden (vgl. Sayers 1989). Nur in diesen beiden Erhebungen, die thematisch der politischen Partizipation gewidmet waren, sind die hier interessierenden unabhängigen und abhängigen Variablen vollständig und anhand gleicher Messinstrumente erhoben worden. Der Datensatz mit einer Größe von N = 3290 enthält nur Westdeutsche ab 21 Jahren, die zwischen 1919 und 1968 geboren wurden. Während Unterschiede zwischen den beiden Messzeitpunkten nicht verfolgt werden sollen, stehen kohortenspezifische Unterschiede im Zentrum des Interesses. Daher werden zwei getrennte Modelle geschätzt: zum einen für die Geburtskohorten 1919-48, die noch nicht von den Bildungsreformen der 1960er Jahre betroffen waren, zum anderen für die später geborenen Geburtskohorten 1949-1968, die von der Bildungsexpansion profitierten.

b) Messinstrumente: Folgende Variablen werden in das Modell integriert: Zur Abbildung der beiden Formen politischer Partizipation als abhängige Variablen wurde jeweils ein Summenindex aus den Antworten auf entsprechende Items gebildet. Der Faktor unkonventionelle politische Partizipation setzt sich zusammen aus „Mitarbeit in Bürgerinitiative“, „an ungenehmigter Demonstration teilgenommen“, „Teilnahme an Besetzungsaktion" und „habe schon einmal politische Gegner eingeschüchtert". Zu den konventionellen Formen politischer Partizipation, die in dieses Modell integriert werden, um das Verhältnis zwischen unkonventioneller und konventioneller Partizipation zu explorieren, gehören die Formen „politische Meinung gesagt“, ,an Wahlen beteiligt“, ,an öffentlicher Diskussion teilgenommen“, „Parteimitarbeit“ und „Kandidaten im Wahl- 
kampf unterstützt“. Erhoben wurde, ob die jeweilige politische Aktivität schon einmal vom Befragten ausgeführt worden ist. Als Bildungsvariable dient der dichotomisierte allgemeinbildende sekundäre Bildungsabschluss (Abitur bzw. Fachabitur versus niedrigere Bildungsabschlüsse), da eine Integration mehrerer Dummy-Variablen in ein Strukturgleichungsmodell nicht sinnvoll ist. Der soziale Status wird über das MPS-Berufsprestige operationalisiert (vgl. Wegener 1988), welches zur Anpassung der Spannweite an die anderen Variablen durch den Wert 40 dividiert wurde. Am unteren Ende dieser Skala finden sich Hilfsarbeiter und Handlanger, das obere Extrem dieser Skala bilden Ärzte. Zur Abbildung der sozialen Herkunft dient das, wiederum durch 40 dividierte, Berufsprestige (MPS) des Vaters. ${ }^{13}$ Die politische Wirksamkeitswahrnehmung, Political Efficacy (PE), wurde über vier Items erhoben, wobei zwei Items der Dimension der „internal political efficacy“ und zwei Items dem Aspekt der „external political efficacy“ zuzurechnen sind (Vetter 1997). ${ }^{14}$ Die interne Konsistenz dieser Skala ist zufriedenstellend (Cronbach's $\alpha=.63 /$ Vier-Item-Skala). Die Variable Politisches Interesse wurde über die Fragestellung erhoben, wie stark sich der oder die Befragte für Politik interessiert und geht als intervallskalierte Variable mit fünf Ausprägungen in die Analyse ein. Die Befragten hatten sich auf einer fünfstufigen Skala zwischen den Extrem-Dimensionen „sehr stark“ und „überhaupt nicht" einzuordnen. ${ }^{15}$ Dieses Instrument entspricht der seit den 1950ern gängigen Messung subjektiven politischen Interesses „by asking people directly how interested they are in politics" (Gabriel und van Deth 1995: 395).

\section{A-P-K-Analyse zur unkonventionellen politischen Partizipation im Zeitverlauf}

Zur adäquaten Untersuchung der Hypothesen zum sozialen Wandel des politischen Interesses und der sozialen Mechanismen (z.B. Bildungsexpansion), die hinter diesem Wandel liegen, wird ein spezifisches A-P-K-Design herangezogen (vgl. Bürklin et al. 1994; Hadjar und Becker 2006a). Grundlage solcher Analysen können Panel-Datensätze oder kumulierte Querschnittsdatensätze über längere Zeitabschnitte sein, auch wenn letztere im Vergleich zu echten, d.h. ereignisorientierten Längsschnittdaten, mit größeren methodisch-statistischen Problemen behaftet sind (Becker 2002). Dem Problem der Konfundierung, das bei gleichzeitiger Schätzung von Alters-, Perioden- und Kohorteneffekten in einem Modell auftritt, wurde in den folgenden Analysen dadurch entgegengewirkt, dass eine der zeitlichen Komponenten durch eine inhaltliche Variable, die mit der entsprechenden Zeit-Variable korrespondiert, ersetzt wurde (vgl. Tuma und Han-

13 Aus datentechnischen Gründen war es nicht möglich, das Berufsprestige der Mutter, das im Zuge zunehmender Frauenerwerbstätigkeit bedeutsamer für die Erfassung der sozialen Herkunft geworden ist, mit zu berücksichtigen.

14 Internal PE: „Ich traue mir zu, in einer Gruppe, die sich mit politischen Fragen befasst, eine aktive Rolle zu übernehmen“, „Die ganze Politik ist so kompliziert, dass jemand wie ich gar nicht versteht, was vorgeht“ (-); external PE: „Die Politiker kümmern sich nicht viel darum, was Leute wie ich denken“ (-), „Leute wie ich haben so oder so keinen Einfluß darauf, was die Regierung tut" (-).

15 Im Jahr 1988 wurde die Variable ausschließlich auf einer zehnstufigen Skala erhoben. Diese Werte wurden durch die einfache Division durch den Wert 2 in ein fünfstufiges Kontinuum überführt. 
nan 1984: 192). Der Wandel unkonventioneller Partizipation wird daher im vollständigen A-P-K-Modell unter Austausch der Kohortenvariable analysiert, d.h. statt des Geburtsjahrs wurde die mit der Bildungsexpansion korrespondierende Variable „Heterogenität" im Hinblick auf die Zusammensetzung der Schülerschaft höherer Schulen nach sozialer Herkunft in das Modell integriert. Eine weitere Maßnahme zur Senkung der Multikollinearität zwischen den Variablen im Rahmen der folgenden Analysen stellt die Verwendung mittelwertszentrierter metrischer Variablen für die Interaktionsterme dar (vgl. Jaccard 2001).

a) Datenbasis: Die Datengrundlage der folgenden Analysen zu den Formen politischer Partizipation bildet ein kumulierter Datensatz der „Allgemeinen Bevölkerungsumfrage der Sozialwissenschaften" (ALLBUS). Dieser aus bevölkerungsrepräsentativen Querschnittserhebungen (1988, 1990, 1991, 1992, 2000) generierte Längsschnitt-Datensatz erlaubt die Bildung synthetischer Kohorten und verfügt über Fallzahlen, die eine detaillierte Analyse der Haupteffekte (Bildung, Status, Kohorte, Periode, Alter, Geschlecht) sowie von Interaktionseffekten (z.B. von Bildung und Kohorte) ermöglichen. ${ }^{16} \mathrm{Um}$ die Kohorten hinsichtlich ihrer Sozialisation bzw. Bildung einheitlich interpretieren zu können, wurden in die Stichprobe zur Analyse der Entwicklung unkonventioneller politischer Partizipation nur Westdeutsche ab 21 Jahren der Geburtsjahre zwischen 1919 bis 1968 aufgenommen. Ostdeutsche oder Ausländer unterscheiden sich in ihren Erfahrungen der politischen Sozialisation signifikant von den Westdeutschen, außerdem liegen für die Ostdeutschen erst ab 1991 Daten vor. Die untere Altersgrenze von 21 Jahren ist notwendig, um eventuelle Effekte durch später erworbene allgemeine sekundäre Bildungszertifikate zu minimieren.

b) Messinstrumente: Abhängige Variable der Analysen ist die unkonventionelle politische Partizipation. Diese dichotomisierte Variable gibt Auskunft darüber, ob der oder die Befragte sich bereits einmal im Rahmen unkonventioneller politischer Partizipationsformen (Unterschriftenaktion, Boykotts, Demonstration oder Bürgerinitiative) ${ }^{17}$, engagiert hat. Das Bildungsniveau, erfragt als das höchste erworbene allgemeine sekundäre Bildungszertifikat, wurde in drei Dummy-Variablen überführt: niedrige Bildung (ohne Abschluss, Hauptschulabschluss), mittlere Bildung (Mittlere Reife) und höhere Bildung (Abitur, Fachabitur). Zur Abbildung von Kohorten wurden aus dem Geburtsjahr mehrere Variablen generiert: Zunächst wurden zur vereinfachten Darstellung der Wand-

16 Eine noch höhere Fallzahl und längere Analysezeiträume wären sicher wünschenswert. Der Längsschnitt kann jedoch nicht ausgeweitet werden, da die benötigten Variablen nur zu diesen Messzeitpunkten erhoben wurden.

17 Es ist darauf hinzuweisen, dass sich diese Operationalisierung der unkonventionellen Partizipation von der Operationalisierung, die in den Analysen des ersten Teils verwendet wurde, unterscheidet. Im Rahmen der Querschnittsanalyse zu den Bestimmungsfaktoren politischer Partizipation gingen die Elemente „ungenehmigte Demonstration“, „Bürgerinitiative“, „Einschüchterung politischer Gegner" und „Besetzungsaktion“ in die Variable der unkonventionellen Partizipation ein. Diese unterschiedlichen Vorgehensweisen ergeben sich aus der mangelhaften Datenlage - die Items wurden nicht kontinuierlich erhoben. Diese Differenzen in der Operationalisierung könnten bei einem Vergleich beider Analysen zu leichten Verzerrungen führen. Da aber als Grundlage der Längsschnittanalyse im A-P-K-Design nur über die Zeit konstant gebliebene Items dienen, stellt sich dieses Problem hier nicht. 
lungsprozesse fünf Kohorten gebildet, die theoretisch voneinander abgrenzbar sind (vgl. Hadjar und Becker 2006a) und jeweils zehn Altersjahrgänge umfassen. Dann wird das Geburtsjahr selbst als mittelwertszentrierte metrische Kohortenvariable in die multivariaten Analysemodelle eingeführt, d.h. von jedem einzelnen Geburtsjahr wurde der Stichproben-Mittelwert der Variable Geburtsjahr abgezogen. In einem letzten Schritt wird zur Minimierung der Probleme der Konfundierung und Multikollinearität bei gleichzeitiger Analyse von Alters-, Perioden- und Kohorteneffekten (Becker 2002) die metrische zeitliche Kohortenvariable durch die inhaltliche Variable "Heterogenität" ersetzt. Diese Variable bietet sich als Kohortenersatz an, weil sie den sozialen Prozess der Bildungsexpansion, für den die Kohorte bzw. das Geburtsjahr als Proxy steht, abbildet, ohne direkt das Bildungsniveau, das in die einzelnen Modelle ebenfalls integriert wird, zu thematisieren. Im Zenit der Bildungsexpansion ist es zu einer zunehmenden Heterogenität der Schülerschaft gekommen, d.h. in dieser Zeit ist die im Vergleich höchste Öffnung der höheren Schulformen für alle soziale Klassen zu konstatieren (Becker 2003). ${ }^{18}$ Zur Bildung der Variable „Heterogenität" wurde für jedes Geburtsjahr das Assoziationsmaß für die Beziehung zwischen sozialer Herkunft (Klassenlage des Vaters nach der Klassifikation von Erikson und Goldthorpe 1992) und Bildungsniveau errechnet. ${ }^{19}$ Jedes einzelne Geburtsjahr wurde dann durch das geburtsjahrspezifische Heterogenitätsmaß (inverses Cramer's V) ersetzt. Die Heterogenität wurde anhand der Klassenzugehörigkeit nach Erikson und Goldthorpe (1992) als Herkunftsmerkmal bestimmt. Eine Alternative wäre das elterliche Bildungsniveau. Vergleichsrechnungen mit sozialer Herkunft nach Bildung der Eltern ergaben jedoch ähnliche Ergebnisse, da Bildungsniveau und soziale Lage hoch korreliert sind. In die A-P-K-Analysen fließen darüber hinaus die metrischen Variablen Alter (in Jahren) und das quadrierte Alter, in Annahme eines nicht-linearen Alterseffekts: Anstieg der Partizipationswahrscheinlichkeit bis um das 60. Lebensjahr, anschließend Stagnation sowie Dummy-Variablen zur Abbildung der Periode (Beobachtungszeitpunkte 1988, 1990, 1991, 1992, 2000) ein. In die Längsschnittanalysen wird zudem das Geschlecht mit seinen dichotomen Ausprägungen ( 0 - weibliches Geschlecht, 1 - männliches Geschlecht) als Kontrollvariable integriert.

\section{Untersuchungsergebnisse}

Zunächst steht die Fragestellung im Zentrum, über welche Einflussfaktoren der Zusammenhang zwischen Bildung und Partizipation verläuft, bevor dann in einem weite-

18 Die Heterogenität der Schülerschaft nach sozialer Herkunft eignet sich deshalb als Kohortenersatz-Variable, da sie, aufgrund der im Zuge der Bildungsexpansion nur langsamen Abnahme der Bildungsungleichheiten (vgl. Becker 2003), nicht im starken Ausmaß mit der Kohortenvariable konfundiert ist und so das Problem der Multikollinearität gesenkt werden kann. Im Vergleich dazu würde das kohortenspezifische Bildungsniveau, z.B. die Abiturientenquote, als Ersatzvariable kaum zu einer spürbaren Entkopplung der unabhängigen Variablen untereinander beitragen, da diese Variable hoch mit dem Geburtsjahr korreliert.

19 Die Heranziehung der väterlichen Schicht ist nur suboptimal, da vor allem in den jüngeren Kohorten die Mütter zunehmend an Bedeutung für die Schichteinstufung der Familie gewinnen. Dennoch sollte die Verzerrung durch diese Vorgehensweise gering sein. 
ren Schritt die Entwicklung unkonventioneller Partizipation im Zeitverlauf anhand der visuellen Inspektion der kohorten-, bildungs- und periodenspezifischen Unterschiede sowie multivariater logistischer Regressionsmodelle untersucht wird.

1. Erklärung konventioneller und unkonventioneller Partizipation:

Ein Strukturgleichungsmodell

In Abbildung 1 sind die in den theoretischen Überlegungen als Hintergrundmechanismen des Zusammenhangs zwischen Bildungsniveau und politischer Partizipation thematisierten Variablen als Mediatoren modelliert. Um der Frage nachzugehen, ob die Bildungsexpansion einen Einfluss auf diese Zusammenhänge hatte, ist ein Strukturgleichungsmodell zur Erklärung konventioneller bzw. unkonventioneller politischer Partizipation für zwei getrennte Gruppen, die früher geborenen Kohorten 1919-48 und für die Geburtsjahrgänge 1949-68, die von der Bildungsexpansion besonders profitiert haben, geschätzt worden. Die Pfadkoeffizienten bzw. erklärten Varianzen für die Kohorten 1919-48 sind dabei in Normalschrift dargestellt, die Parameter für die Kohorten 1949-68 kursiv darunter. Das Strukturgleichungsmodell besitzt eine hohe Anpassungsgüte, wie in den Goodness-of-Fit-Indices (vgl. Hu und Bentler 1999) ersichtlich ist, d.h., dass das hypothetische Modell eine gute Beschreibung der empirischen Wirklichkeit (Datenlage) bietet (vgl. Abbildung 1).

Es finden sich zunächst Belege für den von Mayer und Blossfeld (1990) theoretisierten Statuszuweisungsprozess: Die soziale Herkunft hat einen positiven Effekt auf das Bildungsniveau, das seinerseits das Berufsprestige beeinflusst. Zudem ist auch ein direkter positiver Effekt zwischen sozialer Herkunft und Berufsprestige ersichtlich. Im Hinblick auf einen Vergleich der beiden Gruppen (Geburtsjahrgänge 1919-48 versus Geburtsjahrgänge 1949-68) erweist sich der Konnex zwischen sozialer Herkunft und Bildung als relativ stabil. ${ }^{20}$ Der Einfluss der Bildung auf das Berufsprestige scheint in den später geborenen Kohorten etwas schwächer zu sein, was aber mit einer bisher mangelnden Umsetzung der Bildung auf dem Arbeitsmarkt infolge des jüngeren Alters dieser Gruppe zu tun haben kann.

Als wesentliche Mechanismen hinter dem Zusammenhang zwischen Bildung und politischer Partizipation erweisen sich die politische Wirksamkeitsüberzeugung (,political efficacy") sowie das politische Interesse. Eine höhere Bildung hat eine höhere internale und externale politische Wirksamkeitsüberzeugung zur Folge, d.h., eine positivere Einschätzung, die Fähigkeiten zur politischen Beteiligung zu besitzen und auch Einfluss auf das politische System nehmen zu können. Neben dem direkten Zusammenhang zwischen Bildung und politischer Wirksamkeitsüberzeugung ist auch ein indirekter, über das Berufsprestige vermittelter Zusammenhang ersichtlich. Höhere Bildung geht mit einem höheren Berufsprestige einher, das seinerseits eine höhere „political effi-

20 Dieser Befund ist unter dem Vorbehalt zu formulieren, dass hier nur zwei Gruppen unterschieden werden. In der später aufgezeigten detaillierten Entwicklung ist sehr wohl eine Tendenz zur Abschwächung des Konnex zwischen sozialer Herkunft und Bildung zu erkennen, wobei der Einfluss der sozialen Herkunft in den jüngsten Geburtsjahrgängen wieder tendenziell zunimmt (vgl. Hadjar und Becker 2006b). 
Abbildung 1: Strukturgleichungsmodell zur Erklärung konventioneller und unkonventioneller politischer Partizipation

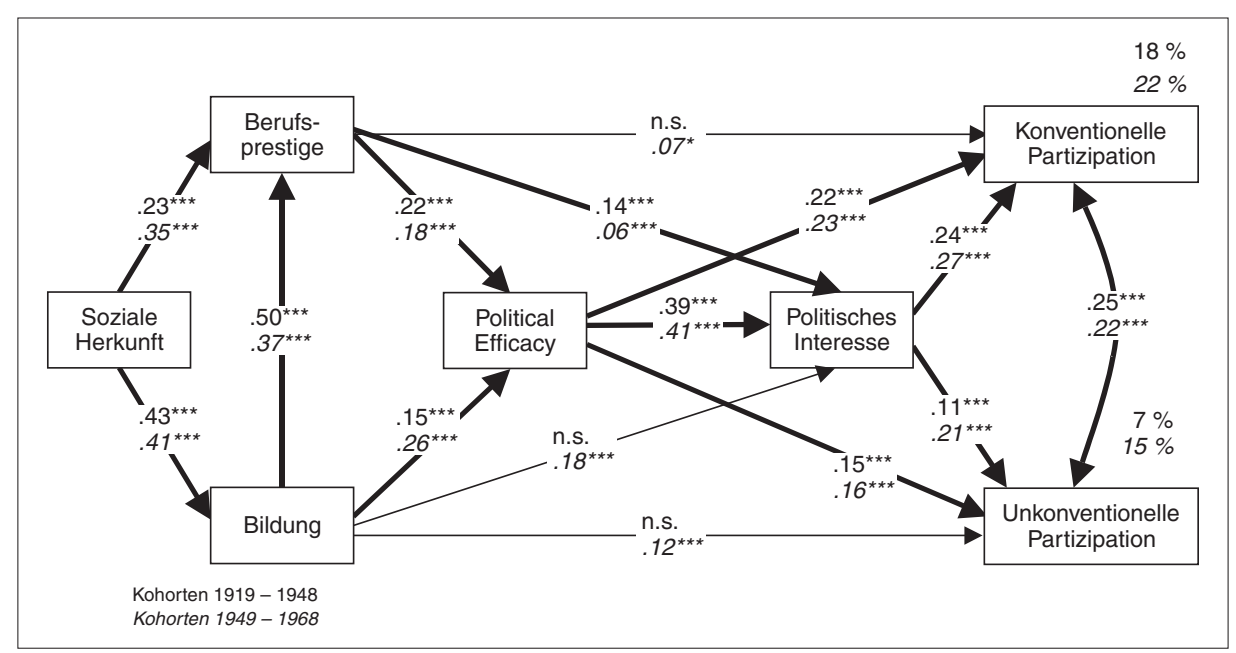

${ }^{* * *} \mathrm{p}<.001,{ }^{* *} \mathrm{p}<.01,{ }^{*} \mathrm{p}<.05 ; \mathrm{N}=2700$, Goodness of Fit-Indices: $\chi^{2}=14.237, \mathrm{df}=12, \mathrm{p}=.286, \mathrm{GFI}=$ $.999, \mathrm{AGFI}=.995, \mathrm{RMR}=.005, \mathrm{RMSEA}=.006, \mathrm{SRMR}=.006$

Quelle: ALLBUS 1988, 1998; eigene Berechnungen.

cacy“ zur Folge hat. Eine höhere politische Wirksamkeitsüberzeugung ist mit einem ausgeprägteren Interesse an Politik verbunden, das sich positiv auf das Ausmaß an konventioneller und unkonventioneller Partizipation auswirkt. $\mathrm{Zu}$ diesem über das politische Interesse mediierten Effekt gibt es jedoch auch direkte Einflüsse der „political efficacy“ auf die konventionelle und die unkonventionelle Partizipation. Wie die positive Korrelation zwischen den beiden Beteiligungstypen zeigt, sind diese Formen politischer Partizipation nicht als Gegensatzpaar anzusehen, sondern stehen in einem positiven Zusammenhang. Wer konventionelle Formen der Partizipation nutzt, ist mit einer bestimmten Wahrscheinlichkeit auch in unkonventionelle Formen involviert. Im Vergleich zwischen den früher geborenen Kohorten und den später geborenen Kohorten fällt auf, dass sich an diesen Mechanismen wenig geändert hat. Interessant ist, dass die direkten und indirekten Effekte der Bildung auf die politische Partizipation eher zuals abgenommen haben (vgl. Abbildung 1).

\section{Politische Partizipation im Zeitverlauf}

Zur abstrahierenden Beschreibung der verschiedenen Geburtskohorten wurden für die visuelle Inspektion der bildungs-, kohorten- und periodenspezifischen Niveaus unkonventioneller Partizipation fünf Kohortenbündel zusammengefasst, die im Folgenden nach ihrem Bildungsniveau gedeutet werden sollen. In Abbildung 2 ist die kohortenspezifische Verteilung der verschiedenen Bildungsniveaus dargestellt, um die Bildungsexpansion über die Kohortenabfolge hinweg sichtbar zu machen. Zusätzlich ist in der Grafik die Heterogenität der Schülerschaft nach sozialer Herkunft abgetragen, d.h., die 
Abbildung 2: Kohortenspezifisches Bildungsniveau und Heterogenität nach sozialer Herkunft

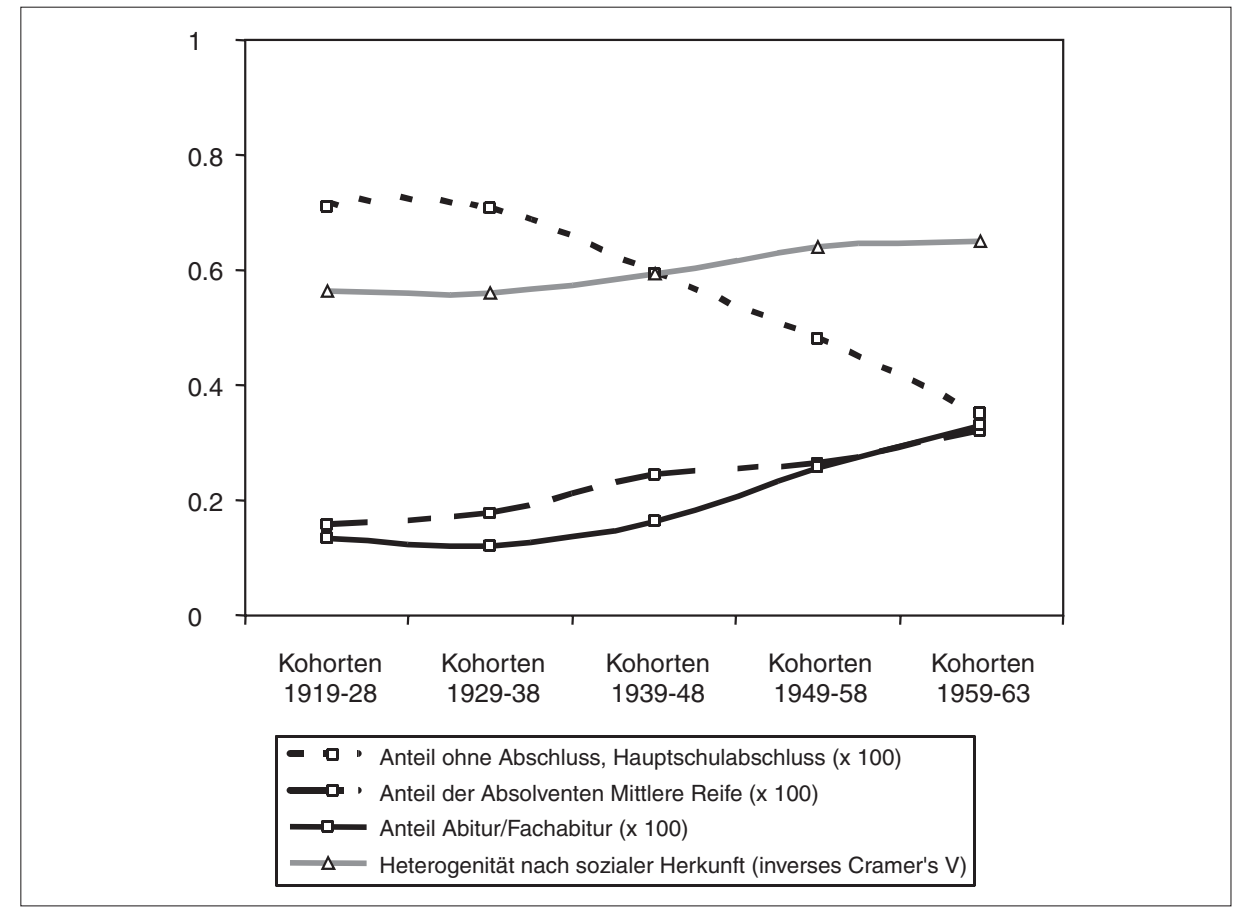

Quelle: ALLBUS 1980-2004; eigene Berechnungen (Transformationsgewichtung).

Entwicklung des Zusammenhangs zwischen sozialer Herkunft (Goldthorpe-Klasse des Vaters) und Bildungsniveau (vgl. Hadjar und Becker 2006a). Darin zeigt sich zunächst eine tendenzielle Ablösung zwischen sozialer Herkunft und Bildungsniveau, die in der jüngeren Kohorte stagniert, weil diese Kohorte aus den Kindern derjenigen besteht, die bereits von der Bildungsexpansion profitierten und somit der Konnex zwischen sozialer Herkunft und Bildung hier wieder enger wird. ${ }^{21}$

Einen ersten Eindruck zu Kohortenunterschieden gibt Abbildung 3. Über alle Messzeitpunkte hinweg finden sich ähnliche Unterschiede. Die ältesten Kohorten zeigen durchgehend die geringste Prävalenz für unkonventionelle Formen politischen Protests, während die junge Generation der zwischen 1959 und 1968 Geborenen bis auf ein Erhebungsjahr zu allen Messzeitpunkten die Spitzenposition einnimmt. Über die Kohortensukzession steigt das Ausmaß unkonventioneller Beteiligungsformen bis zur jüngsten Kohorte hin an. Anfang der 1990er Jahre und zum Jahr 2000 hin sind im Hinblick auf Periodeneinflüsse über alle Kohorten hinweg im Vergleich besonders hohe Ausprägungen an unkonventioneller politischer Partizipation festzustellen.

21 Um eine breite Datenbasis mit größtmöglichen Zellbesetzungen zu erhalten, wurde zur Berechnung der kumulierte ALLBUS 1988-2004 (12 Erhebungen) herangezogen. 
Abbildung 3: Kohortenspezifische Entwicklung unkonventioneller Partizipation

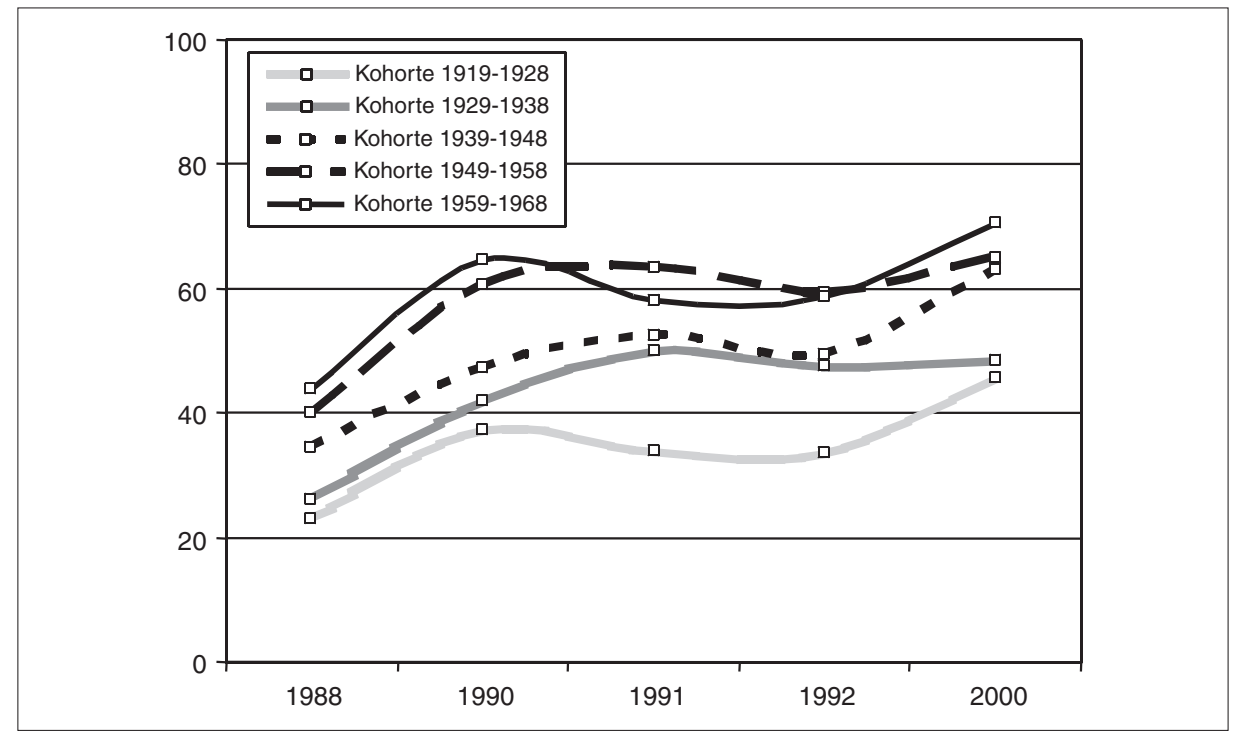

Prozentualer Anteil derjenigen, die bereits eine Form unkonventioneller Partizipation genutzt haben. Quelle: ALLBUS 1988, 1990. 1991, 1992, 2000; eigene Berechnungen (Transformationsgewichtung).

Abbildung 4: Bildungs- und kohortenspezifische unkonventionelle Partizipation

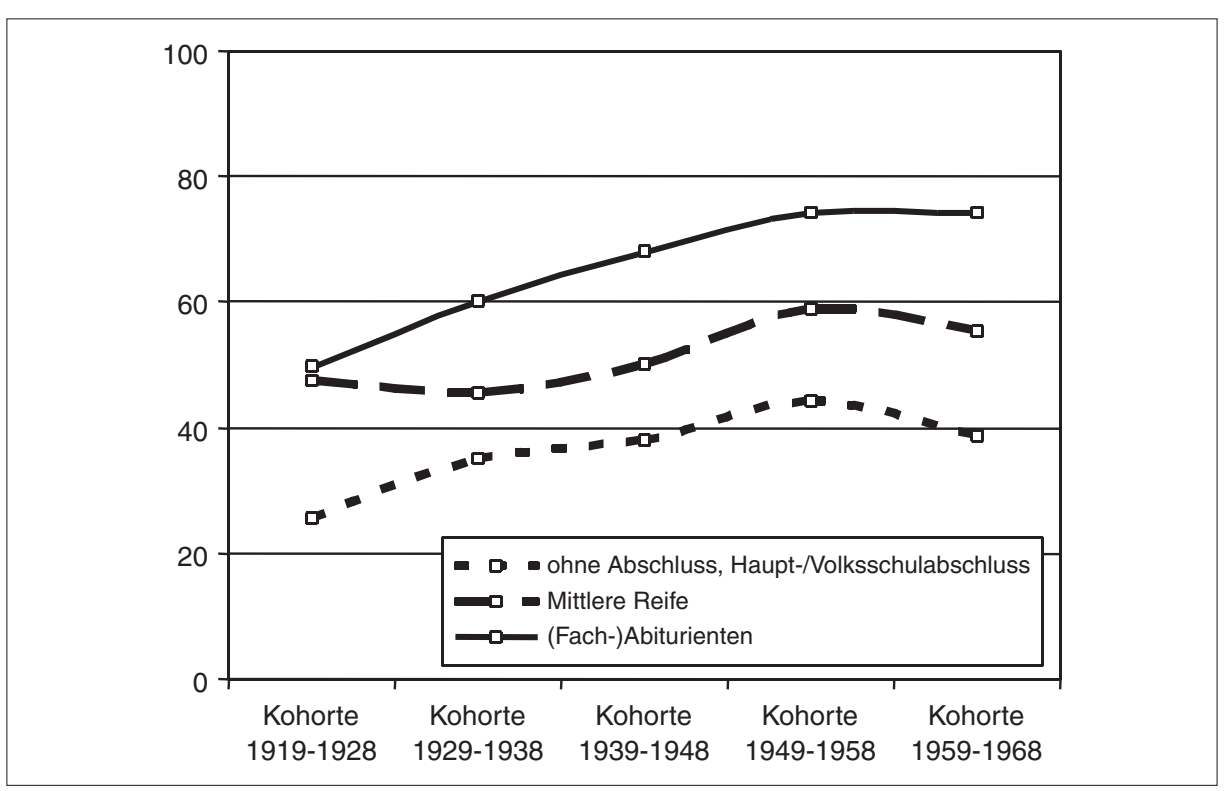

Prozentualer Anteil derjenigen, die bereits eine Form unkonventioneller Partizipation genutzt haben. Quelle: ALLBUS 1988, 1990, 1991, 1992, 2000; eigene Berechnungen (Transformationsgewichtung). 
Die Interaktion zwischen Bildungsniveau und Kohortenzugehörigkeit, die sich auf Entwicklungen im Zuge der Bildungsexpansion bezieht, ist in Abbildung 4 dargestellt. Es sind zunächst markante Bildungsunterschiede zu konstatieren. Offenbar sind die Hochgebildeten die Träger unkonventioneller politischer Beteiligungsformen. Personen mit mittlerer Reife und ehemalige Hauptschulabsolventen sowie Personen ohne Schulabschluss haben eine sehr viel geringere Prävalenz an unkonventioneller Beteiligung. Interessant ist, dass die niedrig Gebildeten ihre negative Distinktion über alle Kohorten beibehalten, während die hoch Gebildeten über die Kohortenabfolge sogar leicht an positiver Distinktion zunehmen. Die Bildungsgruppe mit mittlerer Reife steht in den früheren Kohorten (1919-1928) in ihrem Niveau an unkonventioneller Beteiligung den Absolventen hoher Bildungseinrichtungen (Gymnasien) noch sehr nah, nimmt dann aber zunehmend eine mittlere Position zwischen hoch und niedrig Gebildeten ein. Insgesamt erscheinen die Abstände zwischen den drei Bildungsniveaus über die Kohortenabfolge und damit über die Bildungsexpansion als relativ stabil.

Die visuelle Inspektion kann nur erste Hinweise auf Veränderungen politischer Variablen über die Bildungsexpansion geben. Ohne Betrachtung des Alterseffekts sind die Ergebnisse zu den Kohortenunterschieden nicht ohne Probleme interpretierbar. Im Folgenden soll daher die unkonventionelle politische Partizipation einer multivariaten Analyse unterzogen werden.

In Tabelle 1 finden sich binär-logistische Regressionsmodelle zur Entwicklung unkonventioneller politischer Partizipation im Zeitverlauf. Zunächst ist ersichtlich, dass Abiturienten bzw. Fachabiturienten mit einer 3,6 mal höheren Chance zu denen gehören, die schon eine Form der unkonventionellen Partizipation genutzt haben als niedrig Gebildete (ohne Abschluss oder mit Haupt-/Volksschulabschluss). Die Bildungsgruppe mit mittlerer Reife liegt dazwischen. Bezüglich der Kohorten zeigt sich eine Zunahme der unkonventionellen politischen Partizipation in der Kohortenabfolge. Gegenüber den zwischen 1919 und 1928 Geborenen weisen die Geburtskohorten 1929-38, 1939-48, 1949-58 und 1959-68 eine höhere, über die Kohorten fast linear ansteigende Wahrscheinlichkeit für unkonventionelle politische Partizipation auf (Modell I). Der Effekt der Kontrollvariable Geschlecht weist darauf hin, dass Männer mit einer größeren Wahrscheinlichkeit unkonventionell politisch partizipieren.

Statt der Kohorten-Dummyvariablen wird die Abfolge der Geburtsjahrgänge in Modell II durch eine metrische Variable, das mittelwertszentrierte Geburtsjahr, repräsentiert, um die über die Kohortenabfolge zu beobachtende Entwicklung zu erfassen. Wie sich im Rahmen der visuellen Inspektion (Abbildung 3) und in Modell I der Tabelle 1 bereits andeutete, ist eine steigende Tendenz unkonventioneller politischer Partizipation über die Kohortensukzession festzustellen. Von besonderem Interesse hinsichtlich der Folgen der Bildungsexpansion sind die Interaktionseffekte aus Bildungsniveau und Kohorte (mittelwertszentriertes Geburtsjahr). Der schwache, positive Interaktionseffekt aus (Fach-)Abitur und Kohorte ist im Hinblick auf die Referenzkategorie, den Interaktionseffekt aus niedriger Bildung und Kohorte bei aller Vorsicht ${ }^{22}$ so zu interpretieren: Über die Kohortenabfolge entfernt sich die höchste Bildungsgruppe tenden-

22 Allerdings ist dieser Effekt nur auf dem 10-Prozent-Niveau signifikant und im Hinblick auf das odds ratio nahe 1 nur sehr vorsichtig zu interpretieren. 
Tabelle 1: Binär-logistische Modelle zur Erklärung unkonventioneller politischer Partizipation

\begin{tabular}{|c|c|c|c|}
\hline odds ratios & Modell I & Modell II & Modell III \\
\hline $\begin{array}{l}\text { Bildung } \\
\text { Ref.: ohne Abschluss, Haupt-/Volksschulabschluss } \\
\text { Mittlere Reife } \\
\text { Abitur/Fachabitur }\end{array}$ & $\begin{array}{l}1.813^{* * *} \\
3.574^{* * *}\end{array}$ & $\begin{array}{l}1.814^{* * *} \\
3.334^{* * *}\end{array}$ & $\begin{array}{l}1.854^{* * *} \\
3.688^{* * *}\end{array}$ \\
\hline $\begin{array}{l}\text { Kohorte } \\
\text { Ref.: } 1919-1928 \\
1929-1938 \\
1939-1948 \\
1949-1958 \\
1959-1968\end{array}$ & $\begin{array}{l}1.453^{* * *} \\
1.682^{* * *} \\
2.275^{* * *} \\
1.998^{* * *}\end{array}$ & & \\
\hline Kohorte (Geburtsjahr) & & $1.015^{* * *}$ & \\
\hline $\begin{array}{l}\text { Interaktionseffekt Bildung und Kohorte } \\
\text { Ref.: ohne Abschluss, Haupt-/Volksschulabschluss und Kohorte } \\
\text { Interaktionseffekt Mittlere Reife und Kohorte } \\
\text { Interaktionseffekt Abitur/Fachabitur und Kohorte } \\
\text { Heterogenität nach sozialer Herkunft (Kohorte) }\end{array}$ & & $\begin{array}{c}.995 \\
1.008^{+}\end{array}$ & .194 \\
\hline $\begin{array}{l}\text { Interaktionseffekt Bildung und Heterogenität (Kohorte) } \\
\text { Ref.: ohne Abschluss, Haupt-/Volksschulabschluss und } \\
\text { Heterogenität (Kohorte) } \\
\text { Interaktionseffekt Mittlere Reife und Heterogenität } \\
\quad \text { (Kohorte) } \\
\text { Interaktionseffekt Abitur/Fachabitur und Heterogenität } \\
\quad \text { (Kohorte) }\end{array}$ & & & $\begin{array}{l}2.138 \\
3.016\end{array}$ \\
\hline $\begin{array}{l}\text { Periode } \\
\text { Ref.: } 1988 \\
1990 \\
1991 \\
1992 \\
2000\end{array}$ & & & $\begin{array}{l}2.086^{* * *} \\
2.282^{* * *} \\
2.255^{* * *} \\
3.959^{* * *}\end{array}$ \\
\hline $\begin{array}{l}\text { Alter } \\
\text { Alter }^{2}\end{array}$ & & & $\begin{array}{r}1.049^{* * *} \\
.999^{* * *}\end{array}$ \\
\hline Geschlecht (männlich) & $1.143^{* *}$ & $1.130^{* *}$ & $1.131^{*}$ \\
\hline Interaktionseffekt Geschlecht und Kohorte & & 1.005 & \\
\hline Interaktionseffekt Geschlecht und Heterogenität (Kohorte) & & & $10.294^{*}$ \\
\hline Konstante & $-1.114^{* * *}$ & $-.562^{* * *}$ & -.816 \\
\hline $\mathrm{N}$ & 8.137 & 8.137 & 8.137 \\
\hline Nagelkerkes $\mathrm{R}^{2}$ (in Prozent) & 11,8 & 11,7 & 16,0 \\
\hline
\end{tabular}

*** $\mathrm{p}<.001,{ }^{* *} \mathrm{p}<.01,{ }^{*} \mathrm{p}<.05,^{+} \mathrm{p}<.10$

Quelle: ALLBUS 1988, 1990, 1991, 1992, 2000; eigene Berechnungen (Transformationsgewichtung).

ziell weiter von der niedrigen Bildungsgruppe. D.h., Individuen mit Abitur bzw. Fachabitur gewinnen etwas an Distinktion gegenüber den niedrig Gebildeten. Die Träger der Zunahme der unkonventionellen politischen Partizipation scheinen somit insbesondere die höher Gebildeten in den jüngeren Kohorten zu sein. Das odds ratio bezüglich der Kontrollvariable Geschlecht weist wiederum auf eine stärkere männliche politische Beteiligung hin. Ein Interaktionseffekt aus Kohorte und Geschlecht, der die Entwick- 
lung der Geschlechterunterschiede in der unkonventionellen politischen Partizipation abbilden soll, erweist sich in diesem Modell als nicht signifikant.

Modell III enthält das komplexe A-P-K-Modell, d.h., hier wurden Alters-, Perioden- und Kohorteneffekte simultan geschätzt, wobei zur Minimierung des Konfundierungs- bzw. Kollinearitätsproblems die geburtsjahrgangsspezifische Heterogenität der Schülerschaft hinsichtlich der sozialen Herkunft als Ersatz für die Kohortenvariable dient. $\mathrm{Zu}$ verweisen ist zunächst auf die stabilen Bildungseffekte. Der Kohorteneffekt, hier repräsentiert durch die über die Bildungsexpansion bzw. Kohortensukzession tendenziell zunehmende Heterogenität der Schülerschaft höherer Bildungseinrichtungen, verliert im komplexen A-P-K-Modell an Signifikanz. Der im Rahmen visueller Inspektionen oder einfacher Analysen aufscheinende Befund, dass die jüngeren Generationen stärker unkonventionell partizipieren, ist nur auf ihr jüngeres Alter, ihre tendenziell höhere Bildung und periodenspezifische Ereignisse bzw. gesellschaftliche Zustände zurückzuführen. Die Interaktionseffekte von Bildung und Heterogenität (Kohorte) sind im komplexen A-P-K-Modell nicht mehr signifikant, wenngleich die odds ratio-Werte weiterhin auf den in Modell II aufgezeigten Effekt hinweisen, dass die höhere Bildungsgruppe tendenziell an Distinktion in der unkonventionellen Partizipation gewinnt. Bei Berücksichtigung aller anderen Effekte ist dieser weitere Ausbau des Abstands in der unkonventionellen Partizipation zur niedrigen Bildungsgruppe aber offenbar nicht groß genug, um statistisch bedeutsam zu sein. Im Hinblick auf den Alterseffekt (Alter, Alter ${ }^{2}$ ) ist der hypothesenkonforme Befund zu konstatieren, dass zunächst die Wahrscheinlichkeit unkonventioneller politischer Partizipation mit zunehmendem Alter ansteigt, ab einem bestimmten Alter dann aber stagniert. ${ }^{23}$ Die Periodeneffekte weisen auf einen Anstieg der unkonventionellen politischen Partizipation in der Gesamtstichprobe über den Erhebungszeitraum zwischen 1988 und 2000 hin. Offenbar sind dies Anzeichen für eine generelle Etablierung dieser Beteiligungsformen in der Bevölkerung, und das über bildungs-, kohorten- und altersspezifische Differenzierungen hinweg, denn die periodischen Effekte sind genuin, d.h., sie zeigen sich unter Kontrolle der anderen Einflussvariablen. Der Geschlechtseffekt erweist sich wiederum als robust; Männer sind wahrscheinlich unkonventioneller aktiv als Frauen. Interessant ist der signifikante Interaktionseffekt aus Geschlecht und Heterogenität (als Ersatz für die Geburtskohorte). Offenbar steigt die unkonventionelle politische Partizipation bei den Männern über die Kohortensukzession ungleich stärker als bei den Frauen, wenn das individuelle Bildungsniveau, die Heterogenität der Schülerschaft als Kohortenmerkmal, das Alter und die Periode im Modell kontrolliert bzw. konstant gehalten werden. ${ }^{24}$

23 Das odds ratio von .999 für den Effekt des quadrierten Alters, d.h. des Interaktionseffekts von Alter und Alter ${ }^{2}$, ist so zu deuten, dass der Anstieg der Wahrscheinlichkeit unkonventioneller politischer Partizipation, der sich im linearen Alterseffekt manifestiert, mit zunehmendem Alter geringer wird und schließlich stagniert.

24 Scheinbar hat es jenseits der Folgen der Bildungsexpansion - höhere Bildungsbeteiligung von Frauen, stärkere politische Beteiligung von Frauen - keinen genuinen Angleichungsprozess in der unkonventionellen politischen Partizipation zwischen den Geschlechtern gegeben. 


\section{Diskussion}

Die vermutete Beziehung zwischen Bildungsniveau und unkonventioneller politischer Partizipation sowie die dahinter stehenden sozialen Mechanismen konnten empirisch plausibilisiert werden: Die Ergebnisse zeigen, entsprechend der Hypothese 1, stabile Bildungseffekte: höher Gebildete sind diejenigen, welche im Untersuchungszeitraum das größte Ausmaß an unkonventioneller politischer Partizipation berichteten. Hinter den Bildungseffekten stehen eine mit dem Bildungsniveau steigende politische Wirksamkeitsüberzeugung und ein entsprechend höheres politisches Interesse, wobei ein Teil des Bildungseffekts auch über den sozialen Status vermittelt wird.

Die Resultate der Längsschnittanalyse zur Verknüpfung von Bildungsexpansion und der Entwicklung unkonventioneller Partizipation (politische Mobilisierung) sind so zu rekapitulieren: Auch wenn in einfachen Mittelwertvergleichen Kohortenunterschiede dergestalt aufscheinen, dass jüngere Kohorten verstärkt Formen unkonventioneller politischer Partizipation nutzen (Hypothese 2a), zeichnet doch die komplexe A-P-K-Analyse ein anderes Bild: Offenbar wird der Kohorteneffekt stark von einem Alters- bzw. Lebenszykluseffekt sowie Periodeneinflüssen überlagert. Mit zunehmendem Alter und zunehmender Position im Lebenszyklus steigt zunächst die Wahrscheinlichkeit unkonventionellen politischen Engagements, um im höheren Lebensalter zu stagnieren. Dieses Ergebnis, das die Hypothese 3 stützt, resultiert zum Teil daraus, dass die Jüngeren, entsprechend ihrer Lebensdauer, weniger Zeit hatten, unkonventionelles Partizipationsverhalten zu akkumulieren. Der im höheren Alter stagnierende Alterseffekt ist damit zu erklären, dass über den Lebenszyklus hinweg bei vielen die Distanz zu Sozialisationsumwelten (vgl. Klingemann und Lass 1995), in denen Formen unkonventioneller Partizipation besonders propagiert werden (z.B. Studentenmilieu), zunimmt. Zudem kann die zunehmende Etablierung im Erwerbsleben mit einer nicht mehr steigenden Wahrscheinlichkeit unkonventioneller Partizipation verbunden sein. So gibt es Berufsgruppen, denen bestimmte Formen unkonventioneller politischer Partizipation sogar verboten sind, so dürfen zum Beispiel Beamte in Deutschland nicht demonstrieren. Ein Prozess der Heterogenisierung im Beteiligungsverhalten, d.h., dass sich das Ausmaß an unkonventioneller politischer Partizipation bei den höher Gebildeten der Ausprägung in niedrigeren Bildungsgruppen annähert, weil die Schülerschaft höherer Schulen heterogener bezüglich der sozialen Herkunft wird, ist nicht festzustellen. Damit sprechen die Befunde gegen die Hypothese 2b. Die positive Distinktion der höher Gebildeten bleibt über die Kohorten erhalten. Über den Untersuchungszeitraum ist im Rahmen der abstrahierten Betrachtung ein tendenzieller Anstieg unkonventioneller politischer Partizipation über alle Bildungs-, Altersgruppen und Kohorten hinweg zu beobachten. Somit wird die Hypothese 4 gestützt, dass Periodeneinflüsse das Ausmaß an unkonventioneller politischer Partizipation beeinflussen. Diese periodenspezifische Zunahme, wenngleich im Rahmen dieser Untersuchung nur für den Zeitraum zwischen 1988 und 2000 untersucht, spricht einerseits für eine Etablierung und Normalisierung unkonventioneller politischer Partizipation, die durch globale gesellschaftliche Sozialisationsmechanismen (z.B. Massenmedien; vgl. Klingemann und Fuchs 1995) begünstigt wurde, andererseits aber sicher auch für gesellschaftliche Problemkonstellationen, die mit starker Unzufriedenheit verbunden waren und erhöhte politische Aktivität in den 
Augen der Partizipierenden notwendig machten (vgl. Klages 1984). Zu nennen wäre hier die erhöhte Arbeitslosigkeit in Gesamtdeutschland. Ein dritter Aspekt dieses periodischen Wandels könnte auch eine über die Zeit ansteigende Überzeugung, mit Formen unkonventioneller politischer Partizipation Wirkung zu erzielen (Stichwort: political efficacy; vgl. Vetter 2000), sein.

Die Analyse des Zeitverlaufs hat gezeigt, dass eine Betrachtung verschiedener Zeitdimensionen unabdingbar ist, um „temporalen Fehlschlüssen“ aus dem Wege zu gehen. In diesem Falle haben sich hinter einem starken Kohorteneinfluss vor allem Lebenszyklus- und Periodeneffekte verborgen. Zeitliche Veränderungen sind diffizil und müssen auch hinsichtlich der sozialen Mechanismen, die hinter bestimmten Zusammenhängen liegen, betrachtet werden.

Aus methodischer Sicht ist ebenso auf verschiedene Grenzen dieser Betrachtung hinzuweisen, die aber vor allem auf die mangelnde Datenlage zurückzuführen sind. Zum einen ist ein Beobachtungszeitraum von nur zwölf Jahren für eine A-P-K-Analyse relativ kurz. Wenngleich zu lange Zeiträume wieder das Problem von Selektionseffekten aufwerfen würden, wäre ein Zeitraum von mindestens 20 Jahren mit jährlichen Messungen wünschenswert gewesen: zum einen wegen der größeren Fallzahlen, die bessere Zellbesetzungen insbesondere bezüglich der Interaktionseffekte garantieren, zum anderen, weil dann die Multikollinearitätsproblematik zwischen Alter, Periode und Kohorte auf ein noch niedrigeres Niveau zurückgedrängt werden könnte. Der Erkenntnisgewinn der Analysen hätte auch gesteigert werden können, wenn alle hier als soziale Mechanismen thematisierten Mediatoren (z.B. Political Efficacy) zu allen Messzeitpunkten erhoben worden wären und so auch diese Bindeglieder zwischen Bildung und politischer Partizipation einer Längsschnittbetrachtung zugeführt hätten werden können. Eine adäquate Analyse zur Bildungsexpansion erscheint erst möglich, wenn die von der Bildungsexpansion partizipierenden Kohorten, also die jüngeren Kohorten, ein hohes Alter erreicht haben und damit ihr gesamter Lebensverlauf in die Analyse einflieBen kann (vgl. Hadjar und Becker 2006b). Zudem ist die abhängige Variable der unkonventionellen Partizipation dahingehend zu problematisieren, dass diese nur abbildet, ob der oder die Befragte bereits einmal unkonventionelle Formen politischer Partizipation genutzt hat. Diese Operationalisierung bringt in Bezug auf die zeitlichen Analysen das Problem mit sich, das sehr häufig bei Retrospektivbefragungen auftritt: Es ist nicht zu spezifizieren, zu welchem Zeitpunkt (z.B. in welchem Alter) die Individuen unkonventionell partizipiert haben. ${ }^{25}$

Inhaltlich ist zu fragen, ob diese Analyse die Bedeutung des Bildungseffekts hinreichend klären konnte. Im Hinblick auf die gleich gerichteten Effekte des sozialen Prestiges und des Bildungsniveaus im Strukturgleichungsmodell kann davon ausgegangen werden, dass der Effekt der Bildung zum Teil, entsprechend der Deutung von Bildung als Humankapital, in den bildungsbedingten besseren Chancen zur Statuserzielung begründet liegt. Der verbleibende genuine Bildungseffekt, der einen starken Einfluss behält, könnte auf die thematisierten höheren kognitiven Fähigkeiten und Handlungskompetenzen zurückzuführen sein. Was bislang nicht angesprochen wurde, ist eine

25 Dieser Einwand betrifft allerdings nicht die im Zentrum der Analysen stehenden Kohorteneffekte, da hier nur die akkumlierte Partizipation und nicht der Zeitpunkt im Lebensverlauf von Interesse ist. 
dritte Deutung des Bildungseffekts, in der die Schule als bedeutsame Sozialisationsinstanz bzw. Instanz der Wertevermittlung fokussiert wird (vgl. Hadjar 2006). Eine zu prüfende Hypothese wäre, ob sich verschiedene Schulformen im Hinblick auf die Propagierung politischer Partizipation voneinander unterscheiden. Dieses Problem zielt vor allem auch auf die Wertefrage, die im Rahmen der Analysen aus Gründen der Komplexitätsreduktion ausgeklammert wurde. So könnte unterstellt werden, dass Gymnasien günstigere Sozialisationsumwelten, zu denen sowohl die von Lehrerinnen und Lehrern vermittelten Inhalte, als auch die Werte der Mitschüler und der Eltern gehören, für postmaterialistische Werte darstellen. ${ }^{26}$ Nach Inglehart (1977) sind Mitbestimmung und Partizipation Kernelemente des postmaterialistischen Wertekosmos. Zu thematisieren ist in diesem Zusammenhang auch die Dauer des Verbleibs im Bildungssystem, aus der sich eine mehr oder weniger starke Ablösung der Individuen von ihren Herkunftsschichten mit ihren spezifischen Werten ergibt. Der Besuch höherer Bildungseinrichtungen bedeutet eine längere Verweildauer, die mit einer stärkeren Sozialisation in partizipationsnahen Umwelten gleichzusetzen ist.

Im Ausblick ergeben sich verschiedene Forschungsfelder für Anschlussuntersuchungen, die aufgrund der Komplexitätsreduktion hier unberücksichtigt geblieben sind. Ein interessanter Aspekt wäre eine weitergehende Analyse des Geschlechtereffekts, der hier bereits als Kontrollvariable diente. Gleichermaßen interessant wäre auch eine Typologisierung politischer Beteiligungsformen nach der Rechts-Links-Einordnung. Während in den 1960er Jahren sicher unkonventionelle Beteiligungsformen hauptsächlich vom „linken Spektrum" genutzt wurden, zeichnete sich in den späteren Entwicklungen auch eine zunehmende Inanspruchnahme dieser Formen der Beteiligung im konservativen oder rechten politischen Spektrum ab (Stichworte: Unterschriftenkampagne gegen Doppelte Staatsbürgerschaft in Hessen, Bürgerinitiativen für den Bau von Autobahnen, Neonazi-Demonstrationen, rechte Hausbesetzungen). Hier könnte gefragt werden, inwieweit im Zuge der Bildungsexpansion auch politische Cleavages in der politischen Beteiligung aufgebrochen worden sind. Ein dritter Ansatzpunkt für weitergehende Untersuchungen ist die Frage der Gründe für politische Partizipation. So spielen sowohl die Zufriedenheit mit dem Regierungshandeln als auch die wahrgenommene Betroffenheit im Hinblick auf bestimmte gesellschaftliche Probleme durch die Bürgerinnen und Bürger eine Rolle für die Entscheidung, ob potenzielle Akteure sich politisch engagieren oder nicht.

Zurückkommend auf die Ausgangsfragestellung, ob im Zuge der Bildungsexpansion eine politische Mobilisierung stattgefunden hat, kann gesagt werden, dass verschiedene Aspekte auf eine solche Mobilisierung hindeuten. Ein höheres Bildungsniveau ist mit einer höheren Wahrscheinlichkeit unkonventioneller politischer Partizipation verbunden; ein höheres kohortenspezifisches Bildungsniveau mit einer stärkeren unkonventionellen politischen Partizipation auf dieser Aggregatebene. Die Prävalenz unkonventioneller politischer Partizipation, die nicht als Ersatz, sondern als Ergänzung konventioneller Beteiligungsformen anzusehen ist, dürfte im Zuge der Bildungsexpansion allein schon deshalb zugenommen haben, weil der Anteil der höher Gebildeten, die, wie aufgezeigt, eine gleichbleibende positive Distinktion in der unkonventionellen politischen

26 Nicht zu vernachlässigen sind hier auch die in Deutschland stark entwickelten Institutionen des beruflichen Bildungssystems (vgl. Lempert 1993). 
Partizipation aufweisen, gestiegen ist. Des Weiteren ist klar ersichtlich, dass über die Kohorten hinweg in allen Bildungsschichten eine Zunahme unkonventioneller politischer Partizipation stattgefunden hat. Offenbar wurden Formen der unkonventionellen Beteiligung auch unter Personen mit niedrigerer Bildung salonfähig, wenngleich zu einem weiterhin geringeren Ausmaß als bei den höher Gebildeten. Dies gibt Anlass, gerade für die Hauptschulen weitere Maßnahmen wie die verstärkte Vermittlung politischer Kompetenzen und politische Bildung anzuregen, damit die niedrig Gebildeten verbleibende große Defizite im Hinblick auf politische Kompetenzen und damit auf Gestaltungsmöglichkeiten weiter aufholen können. Alles in allem ist in Bezug auf die untersuchten Kohorten der zwischen 1919 und 1968 Geborenen nicht von zunehmender Politikverdrossenheit und Desinteresse zu sprechen. Dahrendorfs Forderung nach Bildung als Bürgerrecht, um eine Gesellschaft der mündigen Bürger zu schaffen, hat sich offenbar tendenziell erfüllt, allerdings erweisen sich die Bildungsunterschiede in der politischen Partizipation als persistent.

\section{Literatur}

Abramson, Paul, 1983: Political Attitudes in America. Formation and Change. San Francisco: Freeman.

Armingeon, Klaus, 2007: Political Participation and Associational Involvement. S. 358-383 in: Jan W. van Deth, José Ramón Montero und Anders Westholm (Hg.), Citizenship and Involvement in European Democracies. A Comparative Analysis. London: Routledge.

Arzheimer, Kai, 2002: Politikverdrossenheit. Bedeutung, Verwendung und empirische Relevanz eines politikwissenschaftlichen Begriffs. Opladen: Westdeutscher Verlag.

Barnes, Samuel, Max Kaase und Klaus Allerbeck, 1979: Political Action: Mass Participation in Five Western Democracies. Beverly Hills, CA: Sage Publications.

Baumert, Jürgen, 1991: Langfristige Auswirkungen der Bildungsexpansion. Unterrichtswissenschaft 19: 333-349.

Baumert, Jürgen, Cordula Artelt, Eckhard Klieme, Michael Neubrand, Manfred Prenzel, Ulrich Schiefele, Wolfgang Schneider, Gundel Schümer, Klaus-Jürgen Tillmann und Manfred Weiß(Hg.), 2003: Pisa 2000 - Ein differenzierter Blick auf die Länder der Bundesrepublik Deutschland. Opladen: Leske + Budrich.

Becker, Gary S., 1974: Human Capital. New York: Columbia University Press.

Becker, Rolf, 1998: Bildung und Lebenserwartung in Deutschland. Eine empirische Längsschnittuntersuchung aus der Lebensverlaufsperspektive. Zeitschrift für Soziologie 27: 133-150.

Becker, Rolf, 2002: Wahlbeteiligung im Lebensverlauf. A-P-K-Analysen für die Bundesrepublik Deutschland in der Zeit von 1953 bis 1987. Kölner Zeitschrift für Soziologie und Sozialpsychologie 54: 246-263.

Becker, Rolf, 2003: Educational Expansion and Persistent Inequalities of Education. Utilizing Subjective Expected Utility Theory to Explain Increasing Participation Rates in Upper Secondary School in the Federal Republic of Germany. European Sociological Review 19: 1-24.

Becker, Rolf, 2004: Voter Turnouts in East and West Germany. German Politics 13: 1-19.

Becker, Rolf, und Anja Mays, 2003: Soziale Herkunft, politische Sozialisation und Wählen im Lebensverlauf. Politische Vierteljahresschrift 44: 19-40.

Breckler, Steven J., 1984: Empirical Validation of Affect, Behavior, and Cognition as Distinct Components of Attitude. Journal of Personality and Social Psychology 47: 1191-1205.

Bürklin, Wilhelm, Markus Klein und Achim Ruß, 1994: Dimensionen des Wertewandels. Eine empirische Längsschnittanalyse zur Dimensionalität und der Wandlungsdynamik gesellschaftlicher Wertorientierungen. Politische Vierteljahresschrift 35: 579-606. 
Campbell, Angus, Gerald Gurin und Warren E. Miller, 1954: The Voter Decides. Evanston, Ill./ White Plains, NY: Row, Peterson and Co.

Dahrendorf, Ralf, 1965: Bildung ist Bürgerrecht. Plädoyer für eine aktive Bildungspolitik. Hamburg: Wegner.

Dalton, Russell J., 1984: Cognitive Mobilization and Partisan Dealignement in Advanced Industrial Democracies. The Journal of Politics 46: 264-284.

Deth, Jan W. van, 1990: Interest in Politics. S. 275-312 in: M. Kent Jennings und Jan van Deth (Hg.), Continuities in Political Action. A Longitudinal Study of Political Orientations in Three Western Democracies. Berlin: de Gruyter.

Deth, Jan W. van, 1997: Formen konventioneller politischer Partizipation. Ein neues Leben alter Dinosaurier? S. 291-319 in: Oscar W. Gabriel (Hg.), Politische Orientierungen und Verhaltensweisen im vereinigten Deutschland. Opladen: Leske + Budrich.

Diekmann, Andreas, 2004: Empirische Sozialforschung. Grundlagen, Methoden, Anwendungen. Reinbek bei Hamburg: Rowohlt.

Erikson, Robert, und John H. Goldthorpe, 1992: The Constant Flux. A Study of Class Mobility in Industrial Societies. Oxford: Clarendon Press.

Fuchs, Dieter, 2000: Demokratie der Beteiligung in der modernen Gesellschaft. Einige demokratietheoretische Überlegungen. S. 250-280 in: Oskar Niedermayer und Bettina Westle (Hg.), Demokratie und Partizipation. Festschrift für Max Kaase. Wiesbaden: Westdeutscher Verlag.

Gabriel, Oscar W., und Jan W. van Deth, 1995: Political Interest. S. 390-411 in: Jan W. van Deth und Elinor Scarbrough (Hg.), The Impact of Values. Oxford.

Hadjar, Andreas, 2006: Bildungsexpansion und Wandel von sozialen Werten. S. 205-230 in: Andreas Hadjar und Rolf Becker (Hg.), Die Bildungsexpansion. Erwartete und unerwartete Folgen. Wiesbaden: VS Verlag für Sozialwissenschaften.

Hadjar, Andreas, und Rolf Becker, 2006a: Bildungsexpansion und Wandel des politischen Interesses in Westdeutschland zwischen 1980 und 2002. Politische Vierteljahresschrift 47: 12-34.

Hadjar, Andreas, und Rolf Becker (Hg.), 2006b: Die Bildungsexpansion. Erwartete und unerwartete Folgen. Wiesbaden: VS Verlag für Sozialwissenschaften.

Hecken, Anna, 2006: Bildungsexpansion und Frauenerwerbstätigkeit. S. 123-155 in: Andreas Hadjar und Rolf Becker (Hg.), Die Bildungsexpansion. Erwartete und unerwartete Folgen. Wiesbaden: VS Verlag für Sozialwissenschaften.

Herz, Thomas A., 1987: Werte, sozio-politische Konflikte und Generationen. Eine Überprüfung der Theorie des Postmaterialismus. Zeitschrift für Soziologie 16: 56-69.

Hoffmann-Lange, Ursula, 1993: Jugend und Politik. S. 247-251 in: Uwe Andersen und Wichard Woyke (Hg.), Handwörterbuch des politischen Systems der Bundesrepublik Deutschland. 4. Auflage. Bonn: Bundeszentrale für Politische Bildung.

$H u$, Li-tze, und Peter B. Bentler, 1999: Cutoff Criteria for Fit Indexes in Covariance Structure Analysis. Conventional Criteria versus New Alternatives. Structural Equation Modeling 6: 1-55.

Inglehart, Ronald, 1977: The Silent Revolution. Changing Values and Political Styles among Western Publics. Princeton: Princeton University Press.

Inglehart, Ronald, 1997: Modernization and Postmodernization. Cultural, Economic, and Political Change in 43 Societies. Princeton, NJ: Princeton University Press.

Jaccard, James, 2001: Interaction Effects in Logistic Regression. Thousend Oaks: Sage.

Jennings, M. Kent, 1996: Political Knowledge over Time and across Generations. Public Opinion Quarterly 60: 228-252.

Kaase, Max, 1984: The Challenge of the „Participatory Revolution“ in Pluralist Democracies. International Political Science Review 5: 299-318.

Kaase, Max, 2000: Politische Beteiligung. S. 473-478 in: Uwe Andersen und Wichard Woyke (Hg.), Handwörterbuch des politischen Systems der Bundesrepublik Deutschland. 4. Auflage. Wiesbaden: Westdeutscher Verlag.

Kaase, Max, und Alan Marsh, 1979: Distribution of Political Action. S. 167-201 in: Samuel H. Barnes, Max Kaase und Klaus Allerbeck (Hg.), Political Action. Mass Participation in Five Western Democracies. Beverly Hills: Sage.

Klages, Helmut, 1984: Wertorientierungen im Wandel. Frankfurt a.M./New York: Campus. 
Klein, Thomas, Rainer Unger und Alexander Schulze, 2006: Bildungsexpansion und Lebenserwartung. S. 311-331 in: Andreas Hadjar und Rolf Becker (Hg.), Die Bildungsexpansion. Erwartete und unerwartete Folgen. Wiesbaden: VS Verlag für Sozialwissenschaften.

Klingemann, Hans-Dieter, und Dieter Fuchs (Hg.), 1995: Citizens and the State. Oxford.

Klingemann, Hans-Dieter, und Jürgen Lass, 1995: Bestimmungsgründe politischer Beteiligung in Ost- und West-Berlin. S. 148-163 in: Hans-Dieter Klingemann, Lutz Erbring und Nils Diederich (Hg.), Zwischen Wende und Wiedervereinigung. Analysen zur politischen Kultur in West- und Ost-Berlin 1990. Opladen: Westdeutscher Verlag.

Kohlberg, Lawrence, 1996: Die Psychologie der Moralentwicklung. Herausgegeben von Wolfgang Althof unter Mitarbeit von Gil Noam und Fritz Oser. Frankfurt a.M.: Suhrkamp.

Kornelius, Bernhard, und Dieter Roth, 2004, Politische Partizipation in Deutschland. Ergebnisse einer repräsentativen Umfrage. Gütersloh: Verlag Bertelsmann Stiftung.

Krebs, Dagmar, und Peter Schmidt, 1993: Political Efficacy: A Cross-National Comparison of Germany, Great Britain, Italy, and the United States. S. 152-166 in: Dagmar Krebs und Peter Schmidt (Hg.), New Directions in Attitude Measurement. Berlin: de Gruyter.

Krimmel, Iris, 2000: Politische Beteiligung in Deutschland - Strukturen und Erklärungsfaktoren. S. 611-639 in: Jürgen W. Falter, Oscar W. Gabriel und Hans Rattinger (Hg.), Wirklich ein Volk? Die politischen Orientierungen von Ost- und Westdeutschen im Vergleich. Opladen: Westdeutscher Verlag.

Leighley, Jan E., 1995: Attitudes, Opportunities and Incentives. A Review Essay on Political Participation. Political Research Quarterly 48: 181-209.

Lengfeld, Holger, Stefan Liebig und Alfredo Märker, 2000: Politisches Engagement, Protest und die Bedeutung sozialer Ungerechtigkeit. Aus Politik und Zeitgeschichte B 7-8: 22-31.

Lempert, Wolfgang, 1993: Moralische Sozialisation im Beruf. Bedingungsvarianten und -konfigurationen, Prozeßstrukturen, Untersuchungsstrategien. Zeitschrift für Sozialisationsforschung und Erziehungssoziologie 13: 2-34.

Lösche, Peter, 1995: Parteienverdrossenheit ohne Ende? Polemik gegen das Lamentieren deutscher Politiker, Journalisten, Politikwissenschaftler und Staatsrechtler. Zeitschrift für Parlamentsfragen 26: 149-159.

Mayer, Karl-Ulrich, und Hans-Peter Blossfeld, 1990: Die gesellschaftliche Konstruktion sozialer Ungleichheit im Lebensverlauf. S. 297-318 in: Peter A. Berger und Stefan Hradil (Hg.), Lebenslagen, Lebensläufe, Lebensstile. Göttingen.

Mayer, Karl-Ulrich, und Johannes Huinink, 1990: Alters-, Perioden-, Kohorteneffekte in der Analyse von Lebensverläufen oder: Lexis ade? S. 442-459 in: Karl-Ulrich Mayer (Hg.), Lebensverläufe und sozialer Wandel. Sonderheft 31 der Kölner Zeitschrift für Soziologie und Sozialpsychologie. Opladen: Westdeutscher Verlag.

Meulemann, Heiner, 1987: Bildung, Generationen und die Konjunkturen des Werts Leistung. Über die Vielschichtigkeit eines Wertewandels. Zeitschrift für Soziologie 16: 272-287.

Milbrath, Lester W., und Madan Lal Goel, 1977: Political Participation. How and Why Do People Get Involved in Politics? 2. Auflage. Chicago: Rand McNally.

Müller, Walter, 1998: Erwartete und unerwartete Folgen der Bildungsexpansion. S. 81-112 in: Jürgen Friedrichs, M. Rainer Lepsius und Karl-Ulrich Mayer (Hg.), Die Diagnosefähigkeit der Soziologie. Sonderheft 38 der Kölner Zeitschrift für Soziologie und Sozialpsychologie. Opladen: Westdeutscher Verlag.

Niedermayer, Oskar, 2001: Bürger und Politik. Politische Orientierungen und Verhaltensweisen der Deutschen. Wiesbaden: Westdeutscher Verlag.

Opp, Karl-Dieter, 1985: Konventionelle und unkonventionelle politische Partizipation. Zeitschrift für Soziologie 14: 282-296.

Sayers, Lois W., 1989: Pooled Time Series Analysis. Thousand Oaks, CA: Sage.

Schelsky, Helmut, 1956: Soziologische Bemerkungen zur Rolle der Schule in unserer Gesellschaftsverfassung. S. 9-50 in: Helmut Schelsky (Hg.), Schule und Erziehung in der industriellen Gesellschaft. Würzburg: Werkbund-Verlag. 
Schmidtchen, Gerhard, und Hans-Martin Uehlinger, 1983: Jugend und Staat. S. 105-437 in: Ulrich Matz und Gerhard Schmidtchen, Gewalt und Legitimität (Analysen zum Terrorismus 4/1, hg. vom Bundesminister des Innern). Opladen: Westdeutscher Verlag.

Solga, Heike, und Sandra Wagner, 2001: Paradoxie der Bildungsexpansion. Die doppelte Benachteiligung von Hauptschülern. Zeitschrift für Erziehungswissenschaft 4: 107-127.

Uehlinger, Hans-Martin, 1988: Politische Partizipation in der Bundesrepublik. Opladen: Westdeutscher Verlag.

Tuma, Nancy Brandon, und Michael T. Hannan, 1984: Social Dynamics. Models and Methods. Orlando: Academic Press.

Verba, Sidney, Kai Lehman Schlozman und Henry E. Brady, 1995: Voice and Equality. Civic Voluntarism in American Politics. Cambridge/London: Harvard University Press.

Vetter, Angelika, 1997: Political Efficacy: Alte und neue Messmodelle im Vergleich. Kölner Zeitschrift für Soziologie und Sozialpsychologie 49: 53-73.

Vetter, Angelika, 2000: Frischer Wind in einer alten Beziehung? Political Efficacy und die Bundestagswahl 1998. S. 79-109 in: Jürgen W. Falter, Oscar W. Gabriel und Hans Rattinger (Hg.), Wirklich ein Volk? Die politischen Orientierungen von Ost- und Westdeutschen im Vergleich. Opladen: Westdeutscher Verlag.

Wegener, Bernd, 1988: Kritik des Prestiges. Opladen: Westdeutscher Verlag.

Westle, Bettina, 2001: Politische Partizipation und Geschlecht. S. 131-168 in: Achim Koch, Martina Wasmer und Peter Schmidt (Hg.), Politische Partizipation in der Bundesrepublik Deutschland. Empirische Befunde und theoretische Entwicklungen. Blickpunkt Gesellschaft 6. Opladen: Leske + Budrich.

Korrespondenzanschriften: Dr. Andreas Hadjar, Abteilung Bildungssoziologie, Universität Bern, Muesmattstraße 27, 3012 Bern, Schweiz

E-Mail: andreas.hadjar@edu.unibe.ch 